\title{
Do unit commitment constraints affect generation expansion planning? A scalable stochastic model
}

\author{
Schwele, Anna; Kazempour, Jalal; Pinson, Pierre
}

\section{Published in:}

Energy Systems

Link to article, DOI:

$10.1007 / \mathrm{s} 12667-018-00321-z$

Publication date:

2020

Document Version

Peer reviewed version

Link back to DTU Orbit

Citation (APA):

Schwele, A., Kazempour, J., \& Pinson, P. (2020). Do unit commitment constraints affect generation expansion planning? A scalable stochastic model. Energy Systems, 11, 247-282. https://doi.org/10.1007/s12667-018$00321-z$

\section{General rights}

Copyright and moral rights for the publications made accessible in the public portal are retained by the authors and/or other copyright owners and it is a condition of accessing publications that users recognise and abide by the legal requirements associated with these rights.

- Users may download and print one copy of any publication from the public portal for the purpose of private study or research.

- You may not further distribute the material or use it for any profit-making activity or commercial gain

- You may freely distribute the URL identifying the publication in the public portal 


\section{Do unit commitment constraints affect generation expansion planning? A scalable stochastic model}

\section{Anna Schwele, Jalal Kazempour \& Pierre Pinson}

\section{Energy Systems}

Optimization, Modeling, Simulation, and Economic Aspects

ISSN 1868-3967

Energy Syst

DOI 10.1007/s12667-018-00321-z

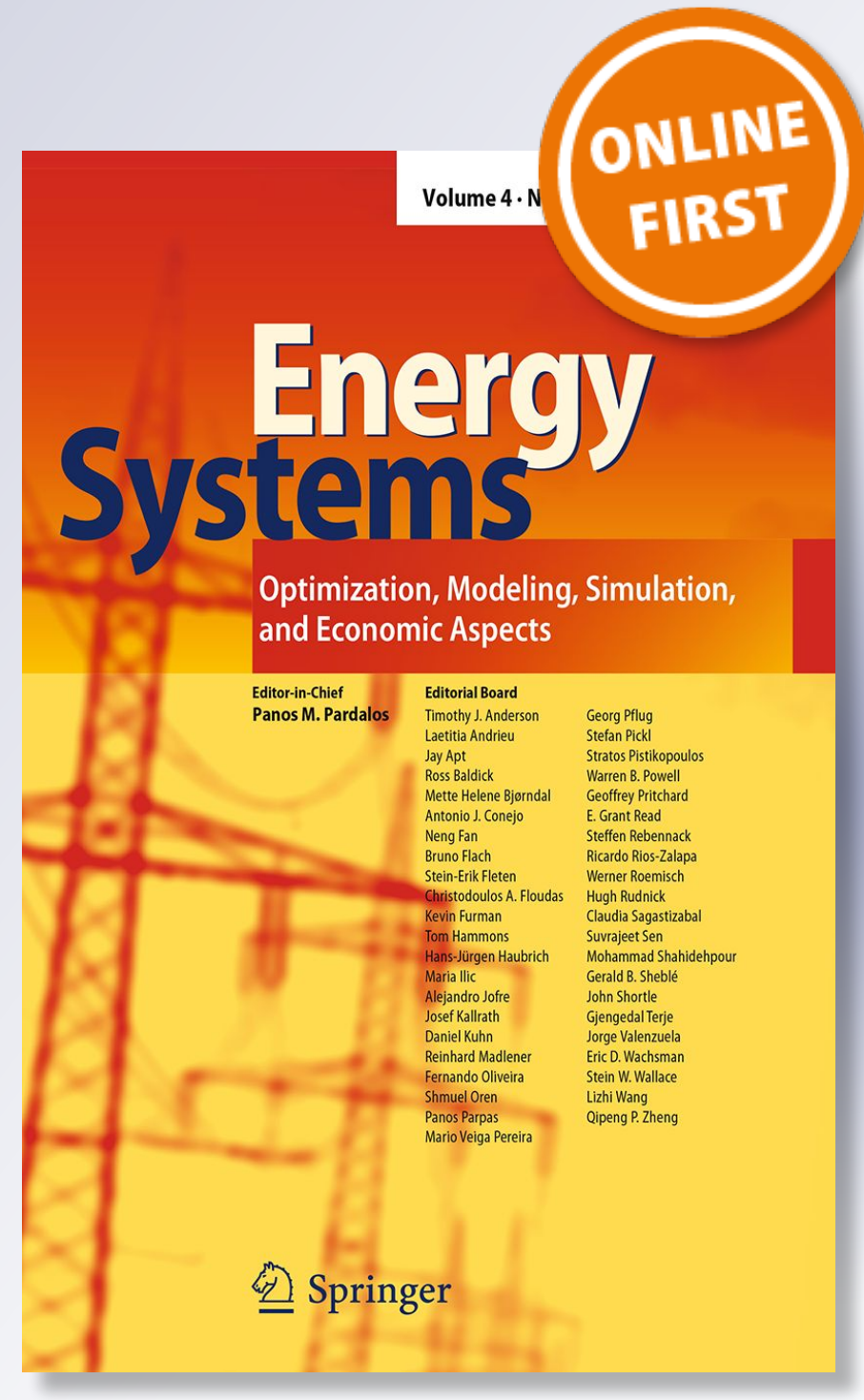

照 Springer 
Your article is protected by copyright and all rights are held exclusively by SpringerVerlag GmbH Germany, part of Springer Nature. This e-offprint is for personal use only and shall not be self-archived in electronic repositories. If you wish to self-archive your article, please use the accepted manuscript version for posting on your own website. You may further deposit the accepted manuscript version in any repository, provided it is only made publicly available 12 months after official publication or later and provided acknowledgement is given to the original source of publication and a link is inserted to the published article on Springer's website. The link must be accompanied by the following text: "The final publication is available at link.springer.com". 


\title{
Do unit commitment constraints affect generation expansion planning? A scalable stochastic model
}

\author{
Anna Schwele ${ }^{1}$ (D) Jalal Kazempour ${ }^{1}$ (D) $\cdot$ Pierre Pinson $^{1}$ (D)
}

Received: 8 January 2018 / Accepted: 17 December 2018

(c) Springer-Verlag GmbH Germany, part of Springer Nature 2019

\begin{abstract}
Due to increasing penetration of stochastic renewable energy sources in electric power systems, the need for flexible resources especially from fast-start conventional generation units (e.g., combined cycle gas turbine plants) is growing. The fast-start conventional units are being operated more frequently in order to respond to the variability and uncertainty of stochastic generation. This raises two important technical questions: as it is common in the literature, is it still an appropriate simplification to ignore the operational unit commitment (UC) constraints of conventional units within the generation expansion planning optimization? And if not, which UC constraint impacts most the expansion planning outcomes? To answer these questions, this paper aims at measuring the planning inefficiency (i.e., the underestimation of need for new generation capacity) caused by ignoring each UC constraint. To this purpose, we develop a centralized network-constrained generation expansion planning model incorporating UC constraints. In particular, we model start-up and shut-down costs, minimum production level and hourly ramping limits of conventional units. Wind power production is considered as the only source of uncertainty, and is modeled through a set of scenarios. A two-stage stochastic programming tool is used, whose first stage determines the long-term expansion and short-term UC decisions over different hours of representative days, while the second stage models the real-time operation for accommodating imbalances arising from wind deviation under different scenarios. Since this problem is potentially hard to solve especially with a large number of representative days and scenarios, a multi-cut Benders' decomposition algorithm is implemented. The well-functioning of the proposed model and the impact of each UC constraint on planning outcomes are evaluated using an extensive numerical study. In our case studies, the exclusion of ramping constraints from planning optimization causes large error and is the most distorting simplification.
\end{abstract}

$凶$ Anna Schwele

schwele@elektro.dtu.dk

Extended author information available on the last page of the article 
Keywords Generation expansion planning (GEP) - Unit commitment

(UC) constraints · Wind power uncertainty - Two-stage stochastic programming .

Mixed-integer linear programming · Multi-cut Benders' decomposition

\section{Introduction}

\subsection{Background and aim}

The share of electricity production from renewable sources is increasing worldwide. Wind power is expected to contribute strongly to electricity generation in the coming decades, despite its inherent variability and uncertainty [20]. In 2015, electricity produced from renewable energy sources contributed more than one quarter $(28.8 \%)$ of the European Union's gross electricity consumption. In particular, more than half $(51.3 \%)$ of annual electricity demand in Denmark in 2015 was supplied by renewable energy sources [2], and this value is expected to reach $100 \%$ by $2050[11,46]$.

The generation expansion planning (GEP) is one of the most important decisionmaking problems in electric power systems, which determines the technology, capacity and location of new generation units to be built. Pursuing simplicity, it is common in the literature to ignore unit commitment (UC) characteristics of the conventional generation units, e.g., ramping limits, start-up and shut-down costs, minimum production level, and minimum up- and down-time constraints, within the GEP problem. However, the conventional generators especially flexible units (e.g., combined cycle gas turbine plants) are being committed and operated more frequently with increasing wind power penetration $[15,41,49]$. The first reason for this is the variability of wind power generation, which makes the dispatch of flexible conventional units quite volatile over time along with short but steep ramps, such that the UC constraints corresponding to those units are often active. The second reason is the uncertainty of wind generation, which requires more operational flexibility from conventional generation units to offset the power imbalance arising from wind forecast error [45]. Due to these reasons, the exclusion of UC constraints from the GEP optimization problem might no longer be an appropriate simplification.

In this paper, we are interested in answering two main technical questions: do UC constraints affect generation expansion planning? And if so, how can we measure the planning inefficiency (i.e., the underestimation of need for new generation capacity) caused by discarding each UC constraint. These power economics questions motivate the following methodological question: how can UC constraints (including binary variables and inter-temporal constraints) as well as wind uncertainty be simulated while ending up in a scalable approach? To answer these questions, we propose a two-stage stochastic program, whose first stage determines the long-term expansion and short-term unit commitment decisions over different hours of representative days. In addition, the second stage models the real-time operations for resolving imbalances due to wind deviation under different wind power scenarios. Since this problem is potentially hard to solve especially with a large number of representative days and scenarios, a multi-cut Benders' decomposition algorithm is implemented. This proposed approach allows decomposing the original mixed-integer linear programming 
(MILP) problem into a mixed-integer linear master problem and a set of continuous linear subproblems, one per scenario and representative day.

\subsection{Literature review and contributions}

The following section analyzes different approaches to GEP models in the literature. This review focuses on modeling techniques used for GEP problems as well as the consideration of operational details such as UC constraints. In general, the capacity expansion problems can be modeled in two ways: static and dynamic (multi-stage) models [8]. In the static approach, all expansion decisions are made at a single point in time, i.e., at the beginning of a target year, representing the whole planning horizon. In contrast, the dynamic (multi-stage) planning model makes expansion decisions at different points in time of the planning horizon, which allows to adapt to future changes in the system and leads to more accurate solutions. However, the complexity of the problem in dynamic model is further increased.

The existing GEP models typically consider a limited level of details on technical constraints of conventional units. For example, reference [18] formulates a multiyear GEP problem using a two-stage stochastic MILP problem, but does not consider network and UC constraints. In [13], a generalized disjunctive programming model for dynamic investment planning in different energy sources, i.e., renewable and nonrenewable, is presented. The model takes start-up, shut-down and general operational costs into account, but neglects all other UC constraints and transmission limits. In [12], a GEP framework is introduced based on a robust optimization, which portrays an optimal investment plan that mitigates inherent planning uncertainties. The future load, investment and production costs are considered as the sources of uncertainty while transmission network and UC constraints are discarded.

In general, the GEP problem can be viewed in two different ways: adopting a centralized approach or considering a market framework. On the one hand, in a vertically integrated power system, a central planner solves a GEP model that results in an optimal operation for the entire electric energy system, and leads to the most efficient supply of demands. This is also known as a command-and-control approach. On the other hand, a market-based approach framework assumes that each electricity producer determines its own generation investment plan as a competitive or a strategic agent with the aim of maximizing expected profit [8]. A market-based network-constrained strategic generation investment model is proposed in [24]. The resulting model is a stochastic mathematical program with equilibrium constraints, which takes into account rival offering and rival investment uncertainties. In addition, [32] compares the optimal GEP outcomes in a centralized approach to those obtained in a market framework considering that the decision-making investor is either a competitive or a strategic price-maker producer. In [35], an investment equilibrium problem in an oligopolistic market including UC constraints is solved using a diagonalization-based heuristic method.

Neglecting operational details in the GEP problem will underrate the technical hurdles, system cost and additional flexibility needs of large shares of renewable generation [59]. The GEP models in the literature that incorporate more operational 
details are presented in the following. In [56], the start-up and shut-down costs of conventional units is added to the traditional merit order stack model for electricity dispatch through a heuristic method. This heuristic has impact on generation units' outputs, electricity prices, and optimal capacity mix. In [52], a linear programming model for long-term power system investment planning is extended with algorithms that build constraints which capture the effects of units' start-up cost, ramping, average output and adequate total system capacity. Reference [17] proposes a two-stage stochastic mixed-integer linear GEP model with long-term uncertainty in wind pattern while incorporating the UC constraints. The first stage models the investment in capacity, while the second stage schedules the scenario-based commitment and dispatch. The UC constraints included in this model are minimum and maximum production level, maximum reserve, ramp-up and -down limits as well as start-up and shut-down costs of conventional units. The work in [20] compares a screening curve methodology with a static investment model that determines the optimal technology mix. Operational constraints such as a periodic maintenance, must-run units, ramp rates and reserve requirements are incorporated into the model. This approach shows a reduced capacity of inflexible generation after including operational constraints to account for net load variability. Furthermore, a sensitivity analysis shows that ramp rates of base-load generation units have a dominant impact on optimal generation mix. Reference [4] extends the screening curves technique to incorporate operation and maintenance costs. In [36], a GEP problem is modeled in a deterministic manner constrained by minimum stable levels, minimum up- and down-times, while considering start-up and shut-down costs. Reference [51] compares an economic dispatch model with and without UC constraints to determine the importance of utilizing UC for GEP of systems with varying amounts of variability. Reference [21] proposes a methodology to integrate short-term responsiveness into a generation technology optimization model. The proposed linear program with static investment decisions for a single-node system includes must-run constraints, ramping limits, and positive and negative balancing requirements for base, mid, and peak load thermal generation technologies. Reference [22] investigates the optimal expansion planning of fast-response generating capacity, in particular gas-fired units, to accommodate the uncertainty of wind generation. A network-constrained GEP model in [22] includes ramping limits, and maximum and minimum up- and down-time constraints. In [25], the UC problem is integrated into a long-term dynamic investment planning framework, resulting in a mixed-integer linear program. The daily energy planning constraints at an hourly level include start-up and shut-down related decisions, i.e., start-up type, minimum up- and down-time, synchronization, soak and desynchronization time constraints, as well as ramping limits and system reserve requirements. Both day-ahead energy and reserve markets are represented, while no uncertainty is included in the model. In [26], a multi-regional, multi-period, mixed-integer linear programming model is presented, combining optimization technique with a Monte Carlo method. This model determines the optimal power capacity installed in each time interval and region as well as the optimal power generation mix including demand response resources. The UC constraints considered are power production limits of thermal plants and reserve margins. Reference [16] introduces a GEP model in which the embedded operational problem is a convex relaxation of the UC problem, i.e., the Lagrangian dual problem, including 
binary commitment decisions, minimum up- and down-time, and ramping constraints, but ignoring network constraints. Moreover, upward spinning reserves are considered, while transmission constraints are neglected. The results in [16] show that ignoring operational flexibility in GEP results in underestimating the need for investment in flexible generation, possibly causing reserve shortage, load shedding, and curtailment of renewable generation. A mixed-integer linear GEP model in [59] studies how increasing operating reserve requirements, driven by increased renewable penetration, impacts the installed generation mix of a power system. This model includes detailed constraints for short-term operation of different generation technologies and detailed operating reserve requirements. Furthermore, in [41], UC constraints are incorporated into a mixed-integer linear GEP optimization model. These UC constraints are ramping limits, start-up and shut-down costs, and operating reserves. Binary commitment variables are grouped into integer block UC by types of power plants into categories. Although wind uncertainty is not modeled, the results suggest that incorporating detailed UC-derived operations into capacity planning models changes the optimal generation mix by more accurately valuing operational flexibility. In addition, [40] identifies that the most effective UC constraints for generation investment decisions are operating reserves and maintenance related constraints. In the proposed MILP, or clustered integer-based modular planning model, the generation units are aggregated into representative plant clusters by technical characteristics. The UC constraints analyzed in this static investment model are maintenance, start-up and shut-down limits. In [42], a computationally efficient GEP model is formulated as a deterministic MILP with embedded integer clustered UC and maintenance constraints for a system with environmental policies. The model in [42] shows that operational flexibility as well as carbon emission constraints change the optimal capacity mix. It also concludes that ignoring the need for flexibility may lead to the system being unable to meet demand and carbon requirements. Reference [47] investigates the impacts of both chronological representation and operational details on planning outcomes for a varying penetration of renewables. In [61], a stochastic decision-making problem based on a real options approach is developed for a single entity to invest in fossil-fueled generation technologies. Reference [30] proposes a two-phase algorithm to solve a multi-area generation and transmission investment planning model. This model includes renewable targets as well as several operating subproblems with ramping constraints. Reference [31] analyzes the impacts of risk aversion on a co-optimized transmission and generation expansion planning problem in a competitive market. The proposed two-stage stochastic programming problem minimizes the weighted average of expected transmission and generation expansion costs, the operational costs, and their conditional value at risk including a direct current (DC) representation of the network. The first stage models the investment in generation and transmission capacity, while the second stage models the economic dispatch under different market and regulatory conditions. Finally, [44] proposes a framework to optimize the long-term investment in new renewable generation capacity while taking into account the hourly dynamics of electricity supply and demand. This framework combines two different energy modeling and planning tools: TIMES is used as a long-term model for optimizing investment in generation capacity, while EnergyPLAN represents a short-term model for optimizing the system operation. These two models interact in an iterative 
process. The results show that the inclusion of dynamics in the modeling methodology changes the investment strategy.

A variety of model characteristics is identified, e.g., inclusion of uncertainties, network representation, form of modeling the investment decisions, representation of market settlements, time horizon, as well as inclusion of energy policies and investment in different types of technologies. This literature review is summarized in Table 1, which highlights and compares the relevant features of some works reported in the literature and the model proposed in this paper.

Considering the works analyzed in the literature review and summarized in Table 1 , the contributions of this paper are as follows: we propose a centralized static GEP model, which incorporates the operational UC details of conventional units such as minimum and maximum production levels, hourly ramping limits and start-up and shut-down costs of generation units. Our contributions are not methodological, as we use existing mathematical techniques to answer important technical questions that arise in practice: do UC constraints affect generation expansion planning? And if so, how can we measure the misestimation of need for new generation capacity caused by discarding each UC constraint. We provide a tool to compare the generation expansion planning decisions with and without enforcing UC constraints, that allows us to evaluate the importance of each of these constraints. This model considers both conventional and wind units as the investment options, and takes into account the network constraints. To improve representation of operational stage, we consider two short-term settlements, including (i) day-ahead UC stage providing on/off commitment and tentative production schedule of units, and (ii) real-time operation stage providing the final production of units. The resulting model is mixed-integer and linear, and considers wind power uncertainty through a set of scenarios. The renewable incentives are considered in the form of renewable portfolio standards (RPS) [19,39,58]. Pursuing scalability, the proposed two-stage stochastic model is decomposed by representative day and scenario using a multi-cut Benders' decomposition algorithm. Finally, an illustrative example and a larger case study are comprehensively analyzed and the results provide a measure of the impacts of each UC constraint on planning outcomes.

\subsection{Paper organization}

The remainder of this paper is organized as follows. Section 2 describes and clarifies the features and assumptions of the proposed model. Section 3 formulates the model as a two-stage stochastic mixed-integer linear problem. In Sect. 4, the decomposition algorithm is described. Section 5 provides comprehensive analyses of two examples. Finally, Sect. 6 provides some relevant conclusions obtained from the study reported in this paper. 


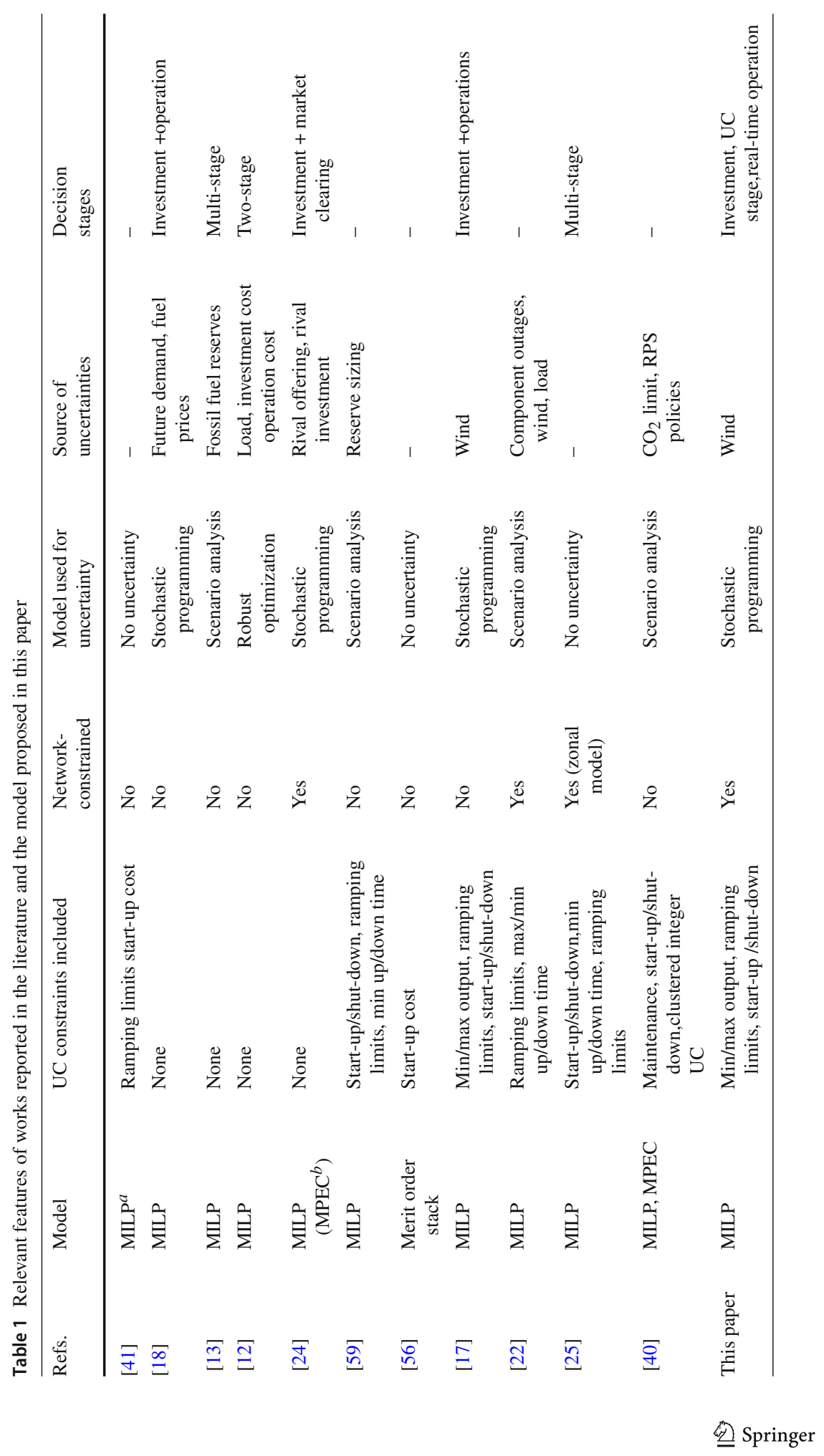




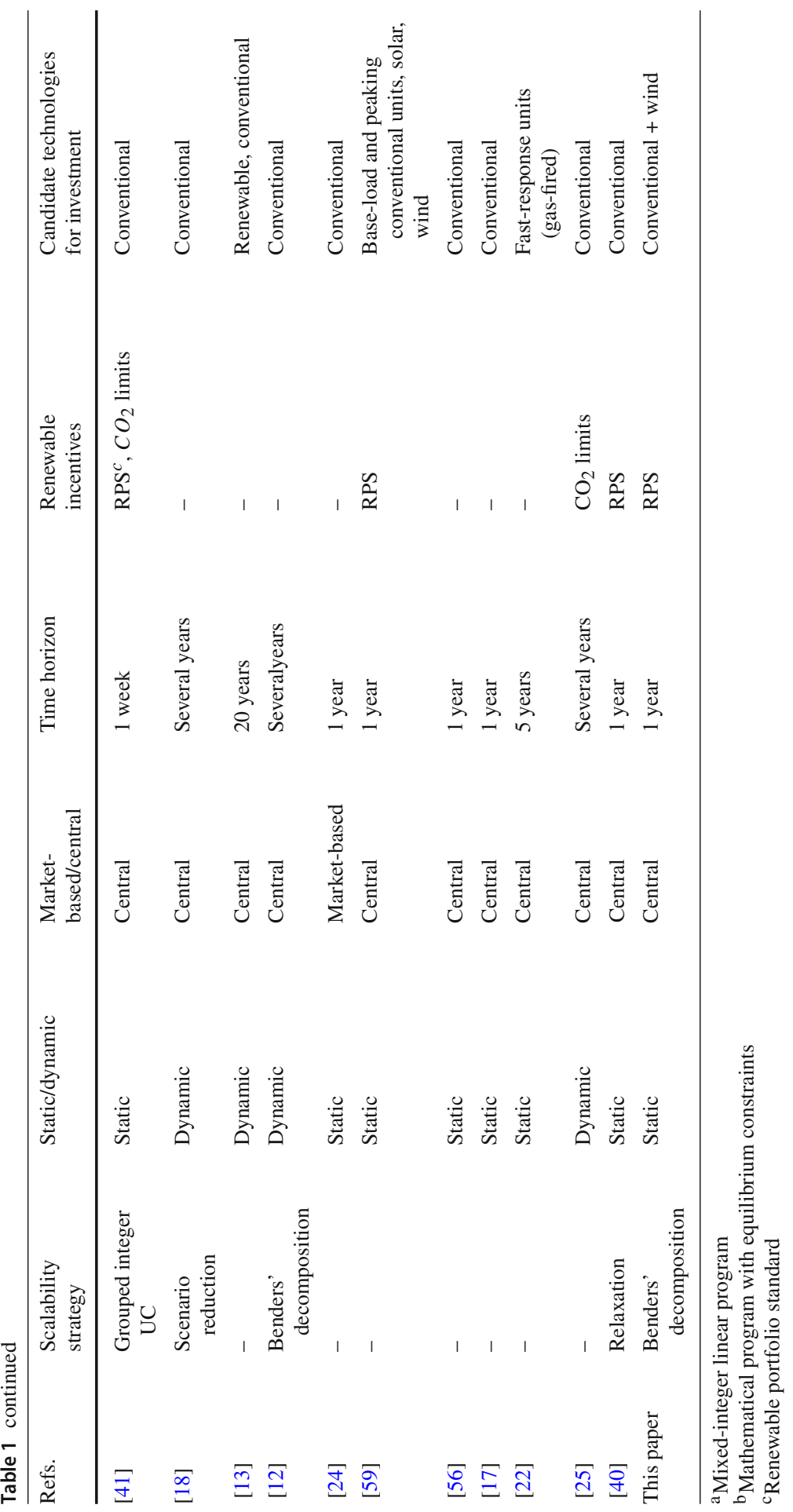




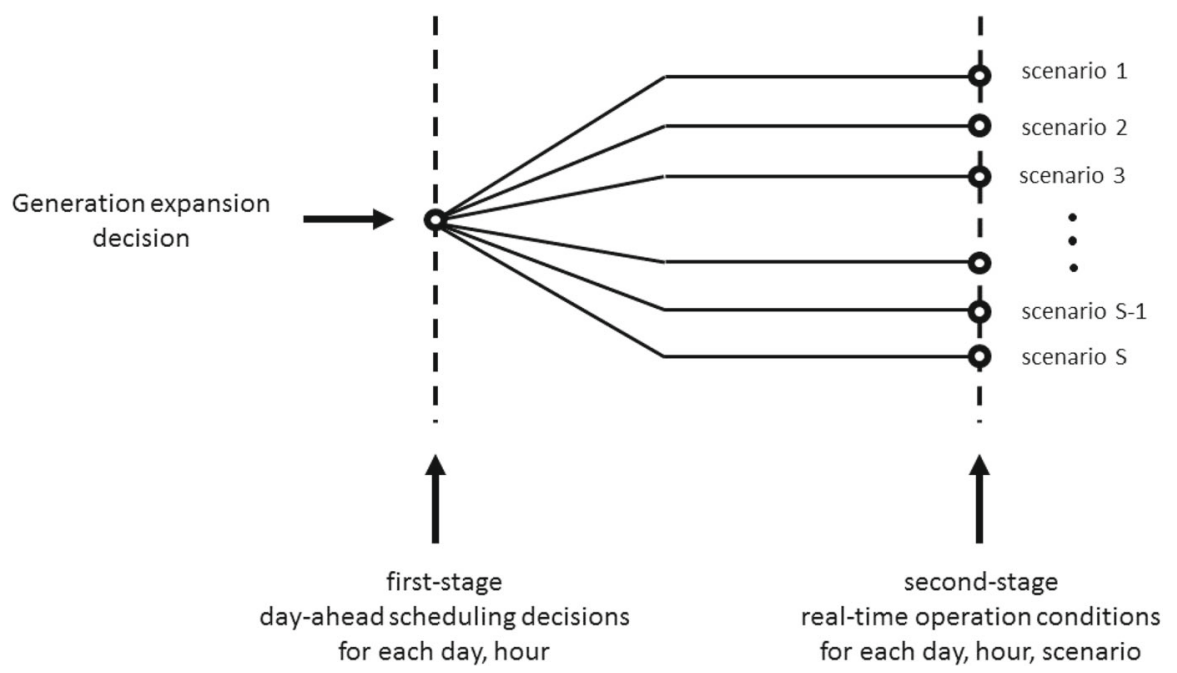

Fig. 1 Two-stage stochastic decision framework of the proposed GEP with UC constraints

\section{Modeling assumptions and features}

\subsection{Framework}

The proposed model is a two-stage stochastic programming problem with fixed recourse. This problem is formulated as a decision tree, see Fig. 1, with each node in the tree representing a decision point. In this two-stage framework, the first stage determines here-and-now decisions prior to the resolution of uncertainty, while the second stage decisions are postponed in a wait-and-see mode after the uncertainties are revealed [9]. The first stage provides the expansion plan as well as daily day-ahead UC and dispatch decisions, while the second stage models the real-time operation to adjust wind power imbalances under each scenario. The wind power uncertainty is represented through a number of discrete realizations, constituting distinct scenarios. In order to make informed investment decisions, the operation of wind-integrated system under all plausible wind scenarios needs to be properly represented.

Taken together, the problem is a two-stage stochastic programming problem, since the first-stage decisions on investment planning and day-ahead scheduling are made under uncertainty of actual wind realization followed later by a second decision in the real-time operation, which allows corrective, i.e., recourse, action to be taken in light of the actual wind power production. All first-stage decisions are made up-front, and cannot be modified as uncertainty about the future is resolved. The objective is to find an optimal decision at the root node that performs well on average under all possible scenarios. 


\subsection{Assumptions}

Following a common approach in the literature, the generation expansion plan is made at a single point in time and establishes the optimal investment for that year, i.e., a static investment plan. A dynamic investment approach would lead to a more complex model. The considered target year includes a set of representative days on an hourly basis. These representative days should be properly selected, such that those days can adequately represent the diversity of daily load and wind conditions over a year, while maintaining the scalability of the model [27,48]. Reference [28] investigates the number of representative periods that are necessary to represent variability in the GEP models.

In this paper, we consider consumers as inelastic loads, thereby instead of social welfare maximization in expectation, we minimize the expected social cost. A central planner, e.g., the system operator, aims at determining the most beneficial expansion plan for the system as a whole, yielding the minimum overall system cost in expectation. We assume the system operator is risk-neutral, but it is straightforward to model risk aversion in a linear fashion [7,31]. The investment costs are annualized and production costs do not change over the planning horizon. The underlying power system is considered to be energy-only, i.e., the producers are paid for their energy production, but not for capacity-based products, e.g., capacity for reserve. In addition, we do not consider decommissioning of existing units by the target year. The wind power production cost is assumed to be zero. The supplied energy is required to meet the demand in each time period within the day-ahead UC stage. In the real-time operation, load shedding and wind curtailment are both allowed, but the former is subject to a high penalty cost for the system.

The investment model is network-constrained; however, during the considered planning horizon, there is no change in the system topology, i.e., the existing transmission lines remain the same throughout the planning horizon and cannot be extended within this model. A study for evaluating the impact of transmission switching on expansion decisions can be found in [60]. A loss-less DC representation of the transmission network is embedded within the investment model, which is linear and appropriate for the GEP. In contrast, an alternative current (AC) representation of transmission system, although more accurate, results in a non-convex model, requiring a convexified GEP model using a relaxation method. The network constraints adequately represent the effect of locating new units at different nodes, i.e., siting of facilities, as well as the impact of transmission congestion. The UC constraints that are regarded and analyzed for their impact on the generation expansion decision include start-up and shut-down costs, minimum production limits, and hourly ramp rate limits of conventional generation units. For the sake of simplicity, active power losses, maintenance and fixed costs, minimum up- and down-times, or fast-start units with the availability of start-up at the real-time operation stage are not included in the proposed model. Including faststart units requires binary variables in the second-stage (real-time stage), which makes the subproblems in our setup mixed-integer, and thus we cannot derive sensitivities anymore for generating Benders' cuts. Therefore, since we need convexity in subproblems, we discard the fast-start units in our model. One alternative decomposition 
solution to consider binary variables in subproblems is Dantzig-Wolfe decomposition [14,54], which has its own pros and cons with respect to Benders' decomposition. A full UC model with all those limits is available in [38]. The only source of uncertainty within the model is wind power generation, which is modeled through a set of finite scenarios. The other potential sources of uncertainty, e.g., assets failure, demand growth, investment cost, and regulatory policies are not considered. However, there is no technical barrier, and they can be modeled using additional scenarios. We aim at investigating the impact of active UC constraints on the optimal generation mix. These UC constraints are often pushed to their limits due to the variability and uncertainty of wind power production. Thus, wind power generation is chosen as the only source of uncertainty.

\section{Proposed model}

The main notation used throughout the paper is defined below:

\section{Indices:}

d Index for representative days.

$l \quad$ Index for loads.

$g \quad$ Index for conventional generation units.

$k \quad$ Index for wind farms.

$(n, m)$ Indices for system nodes.

$s \quad$ Index for wind power scenarios.

$h \quad$ Index for hours.

$o \quad$ Index for generation expansion options.

$(i, j)$ Indices for Benders' iterations.

\section{Sets:}

$\Xi \quad$ Set of optimization variables.

$\mathcal{L} \quad$ Set of loads.

$\mathcal{G} \quad$ Set of all candidate and existing conventional generation units $\left(\mathcal{G}^{\mathrm{E}} \cup \mathcal{G}^{\mathrm{C}}\right)$.

$\mathcal{G}^{\mathrm{C}} \quad$ Set of candidate conventional generation units.

$\mathcal{G}^{\mathrm{E}} \quad$ Set of existing conventional generation units.

$\mathcal{K} \quad$ Set of all candidate and existing wind farms $\left(\mathcal{K}^{\mathrm{E}} \cup \mathcal{K}^{\mathrm{C}}\right)$.

$\mathcal{K}^{\mathrm{C}} \quad$ Set of candidate wind farms.

$\mathcal{K}^{\mathrm{E}} \quad$ Set of existing wind farms.

$\Omega_{n} \quad$ Set of nodes adjacent to node $n$.

Note that sets $\mathcal{G}, \mathcal{K}$ and $\mathcal{L}$ include subscript $n$ if referring to the set of generation units, wind farms and loads, respectively, located at node $n$.

\section{First-stage continuous variables:}

$\bar{x}_{g} \quad$ Capacity of candidate conventional unit $g$ (MW).

$x_{k} \quad$ Capacity of candidate wind farm $k(\mathrm{MW})$.

$\underline{x}_{g} \quad$ Minimum power output of candidate conventional unit $g(\mathrm{MW})$.

$c_{g, d, h}^{\mathrm{SU}} \quad$ Start-up cost of conventional unit $g$ in hour $h$ of day $d(€)$. 
$c_{g, d, h}^{\mathrm{SD}} \quad$ Shut-down cost of conventional unit $g$ in hour $h$ of day $d(€)$.

$p_{g, d, h}^{\mathrm{DA}} \quad$ Power scheduled for conventional unit $g$ in hour $h$ of day $d$ (MW).

$w_{k, d, h}^{\text {DA }} \quad$ Power scheduled for wind farm $k$ in hour $h$ of day $d(\mathrm{MW})$.

$\theta_{n, d, h}^{\text {DA }} \quad$ Voltage angle at node $n$ in hour $h$ of day $d$ at the scheduling stage (rad).

$\underline{\Phi}_{g, d, h}$ An auxiliary continuous variable equal to a bilinear product of $\underline{x}_{g}$ and $u_{g, d, h}$ (MW).

$\bar{\Phi}_{g, d, h}$ An auxiliary continuous variable equal to a bilinear product of $\bar{x}_{g}$ and $u_{g, d, h}$ (MW).

\section{Second-stage continuous variables:}

$p_{l, d, h, s}^{\mathrm{SH}}$ Involuntarily active load shedding of load $l$ in day $d$, hour $h$ under scenario $s$ (MW).

$r_{g, d, h, s}$ Power adjustment of conventional unit $g$ in day $d$, hour $h$ under scenario $s$ (MW).

$w_{k, d, h, s}^{\mathrm{SP}}$ Wind power spillage of farm $k$ in day $d$, hour $h$ under scenario $s$ (MW).

$\theta_{n, d, h, s}^{\mathrm{RT}} \quad$ Voltage angle at node $n$ in day $d$, hour $h$ under scenario $s$ (rad).

$\alpha_{s}^{(i)} \quad$ Benders' proxy variable under scenario $s(€)$

\section{First-stage binary variables:}

$u_{g, d, h} \quad 0 / 1$ variable that is equal to 1 if conventional unit $g$ is scheduled to be committed in day $d$ and in hour $h$.

$u_{g, o}^{\text {opt }} \quad 0 / 1$ variable that is equal to 1 if candidate conventional unit $g$ selects generation expansion option $o$.

\section{Parameters:}

$R P S \quad$ A non-negative factor indicating renewable portfolio standard as target percentage of renewables in future generation mix.

$S E C \quad$ A non-negative factor indicating supply security with respect to system load.

$\bar{L} \quad$ Maximum load.

$\rho_{s} \quad$ Probability of scenario $s$. Note that $\sum_{s} \rho_{s}=1$.

$\sigma_{d} \quad$ Weight of representative day $d$ within the target year. Note that $\sum_{d} \sigma_{d}=365$.

$\lambda_{g}^{\mathrm{SU}} \quad$ Start-up cost of conventional unit $g(€)$.

$\lambda_{g}^{\mathrm{SD}} \quad$ Shut-down cost of conventional unit $g(€)$.

$I_{g} \quad$ Annualized investment cost of candidate conventional unit $g$ (€/MW-year).

$I_{k} \quad$ Annualized investment cost of candidate wind farm $k$ (€/MW-year).

$\bar{X}_{g, o}^{\text {opt }} \quad$ Capacity of expansion option $o$ for candidate conventional unit $g(\mathrm{MW})$.

$\bar{X}_{k} \quad$ Capacity of existing wind farm $k$ (MW).

$\underline{X}_{g, o}^{\text {opt }} \quad$ Minimum power output of candidate conventional unit $g$ under expansion option $o$ (MW).

$C_{g} \quad$ Marginal cost of conventional unit $g(€ / \mathrm{MWh})$.

$L_{l, d, h} \quad$ Power consumption of load $l$ in day $d$ and hour $h$ (MW).

$\bar{P}_{g} \quad$ Capacity of existing conventional unit $g$ (MW).

$\underline{P}_{g} \quad$ Minimum power output of existing conventional unit $g(\mathrm{MW})$. 
$P_{g, d}^{\text {ini }} \quad$ Initial active power output of conventional unit $g$ in day $d(\mathrm{MW})$.

$R_{g}^{+} \quad$ Ramp-up limit of existing conventional unit $g(\mathrm{MW} / \mathrm{h})$.

$R_{g}^{-} \quad$ Ramp-down limit of existing conventional unit $g(\mathrm{MW} / \mathrm{h})$.

$R_{g}^{\text {opt+ }} \quad$ Ramp-up limit of candidate conventional unit $g(\mathrm{MW} / \mathrm{h})$.

$R_{g}^{\text {opt}-} \quad$ Ramp-down limit of candidate conventional unit $g(\mathrm{MW} / \mathrm{h})$.

$R_{g, d}^{\text {ini }} \quad$ Initial adjustment power of conventional unit $g$ in day $d(\mathrm{MW})$.

$F_{n, m} \quad$ Capacity of transmission line $(n, m)(\mathrm{MW})$.

$U_{g, d}^{\text {ini }} \quad$ Initial commitment status of conventional unit $g$ in day $d(0 / 1)$.

$V_{l}^{\mathrm{SH}} \quad$ Value of lost load for load $l$ (€/MWh).

$W_{k, d, h, s}$ Wind power realization (in per unit) of farm $k$ in day $d$, hour $h$ under scenario

$\bar{W}_{k, d, h}^{\mathrm{DA}} \quad \begin{aligned} & s . \\ & \text { Maximum wind power factor (in per unit) of farm } k \text { in day } d \text { and hour } h \text { that }\end{aligned}$ can be scheduled at the scheduling stage. It is assumed to be equal to the expected power production, i.e., $\bar{W}_{k, d, h}^{\mathrm{DA}}=\sum_{s} \rho_{s} W_{k, d, h, s}, \forall k, \forall d, \forall h$.

$X_{n, m}^{\text {line }} \quad$ Reactance of transmission line $(n, m)(\Omega)$.

$M \quad$ A large enough positive value, e.g., maximum capacity of expansion options.

The proposed two-stage stochastic GEP model with UC constraints is formulated as (1)-(4). The objective function (1) minimizes the expected system cost, and is subject to the generation expansion constraints (2), the first-stage constraints (3) and the second-stage constraints (4). The optimization variables of the GEP (1)-(4) are the elements of the set $\Xi^{\mathrm{GEP}}=\left\{u_{g, o}^{\mathrm{opt}}, \underline{x}_{g}, \bar{x}_{g}, x_{k}, c_{g, d, h}^{\mathrm{SU}}, c_{g, d, h}^{\mathrm{SD}}, u_{g, d, h}, p_{g, d, h}^{\mathrm{DA}}, w_{k, d, h}^{\mathrm{DA}}, \theta_{n, d, h}^{\mathrm{DA}}\right.$, $\left.r_{g, d, h, s}, w_{k, d, h, s}^{\mathrm{SP}}, p_{l, d, h, s}^{\mathrm{SH}}, \theta_{n, d, h, s}^{\mathrm{RT}}\right\}$. The objective function is given by (1) below:

$$
\begin{aligned}
\underset{\Xi \mathrm{GEP}}{\operatorname{Minimize}} \sum_{g \in \mathcal{G}^{\mathrm{C}}} I_{g} \bar{x}_{g}+\sum_{k \in \mathcal{K}^{\mathrm{C}}} I_{k} x_{k} \\
+\sum_{d} \sigma_{d}\left[\sum_{(g \in \mathcal{G}), h}\left(c_{g, d, h}^{\mathrm{SU}}+c_{g, d, h}^{\mathrm{SD}}+C_{g} p_{g, d, h}^{\mathrm{DA}}\right)\right. \\
\left.+\sum_{s} \rho_{s}\left(\sum_{(g \in \mathcal{G}), h} C_{g} r_{g, d, h, s}+\sum_{(l \in \mathcal{L}), h} V_{l}^{\mathrm{SH}} p_{l, d, h, s}^{\mathrm{SH}}\right)\right] .
\end{aligned}
$$

The objective function (1) contains the generation expansion cost of candidate units (first line), the system cost in day-ahead stage (second line), and the expected imbalance cost in real-time operations (third line). The first term represents the investment cost of newly built candidate conventional generation units, while the second term refers to those costs for candidate wind farms. The next three terms correspond to the startup and shut-down costs of the conventional generation units and their production cost dispatched in the day-ahead stage. The last two terms represent the expected regulating power cost, i.e., the adjustment in power production, and load shedding cost for the real-time operation. The generation expansion constraints are given by (2) below: 


$$
\begin{aligned}
& \bar{x}_{g}=\sum_{o} u_{g, o}^{\mathrm{opt}} \bar{X}_{g, o}^{\mathrm{opt}}, \quad \forall g \in \mathcal{G}^{\mathrm{C}} \\
& \underline{x}_{g}=\sum_{o} u_{g, o}^{\mathrm{opt}} \underline{X}_{g, o}^{\mathrm{opt}}, \quad \forall g \in \mathcal{G}^{\mathrm{C}} \\
& \sum_{o} u_{g, o}^{\mathrm{opt}}=1, \quad \forall g \in \mathcal{G}^{\mathrm{C}} \\
& x_{k} \geq 0, \quad \forall k \in \mathcal{K}^{\mathrm{C}} \\
& \sum_{k \in \mathcal{K}^{C}} x_{k}+\sum_{k \in \mathcal{K}^{E}} \overline{X_{k}}+\sum_{g \in \mathcal{G}^{C}} \overline{x_{g}}+\sum_{g \in \mathcal{G}^{E}} \overline{P_{g}} \geq S E C \bar{L}, \\
& \sum_{k \in \mathcal{K}^{C}} x_{k}+\sum_{k \in \mathcal{K}^{E}} \overline{X_{k}} \geq R P S\left(\sum_{g \in \mathcal{G}^{C}} \overline{x_{g}}+\sum_{g \in \mathcal{G}^{E}} \overline{P_{g}}+\sum_{k \in \mathcal{K}^{C}} x_{k}+\sum_{k \in \mathcal{K}^{E}} \overline{X_{k}}\right) .
\end{aligned}
$$

Constraints (2a) and (2b) set the capacity and the minimum power output of each candidate conventional generation unit to the pre-specified values in the selected expansion option. Equations (2c) ensure that only one option is selected for each candidate conventional unit. Note that different generation expansion options are considered as inputs, available for a candidate conventional unit $g \in \mathcal{G}^{\mathrm{C}}$ to be located at a given candidate bus $n$, including respective annualized investment cost $I_{g}$, production cost $C_{g}$, capacity $\bar{X}_{g, o}^{\mathrm{opt}}$, minimum output $\underline{X}_{g, o}^{\mathrm{opt}}$, and ramp rate limits $R_{g, o}^{\mathrm{opt}+}$ and $R_{g, o}^{\text {opt- }}$. Notably, the option of no investment needs to be considered with $\bar{X}_{g, o}^{\text {opt }}=0$ $\mathrm{MW}, \underline{X}_{g, o}^{\mathrm{opt}}=0 \mathrm{MW}, R_{g, o}^{\mathrm{opt}+}=0 \mathrm{MW} / \mathrm{h}$, and $R_{g, o}^{\mathrm{opt}-}=0 \mathrm{MW} / \mathrm{h}$. According to (2d), the investment in additional wind capacity is non-negative and continuous. The long-term capacity balance constraint in (2e) requires the total generation capacity to be equal to or greater than a factor of the maximum system load. Constraint (2f) ensures a generation mix according to the given renewable portfolio standard policy, adjusted by the non-negative factor $R P S$. This renewable target constraint imposes a minimum amount of installed power capacity from renewable generation units, defined as a fraction of the system's total capacity. The other potential implementations of RPS-based policy, e.g., in terms of energy rather than capacity, is possible and straightforward. The first-stage constraints are given by (3) below:

$$
\begin{aligned}
& \sum_{g \in \mathcal{G}_{n}} p_{g, d, h}^{\mathrm{DA}}+\sum_{k \in \mathcal{K}_{n}} w_{k, d, h}^{\mathrm{DA}}-\sum_{l \in \mathcal{L}_{n}} L_{l, d, h}=\sum_{m \in \Omega_{n}} \frac{1}{X_{n, m}^{\mathrm{line}}}\left(\theta_{n, d, h}^{\mathrm{DA}}-\theta_{m, d, h}^{\mathrm{DA}}\right), \\
& \quad \forall n, \forall d, \forall h \\
& \underline{x}_{g} u_{g, d, h} \leq p_{g, d, h}^{\mathrm{DA}} \leq \bar{x}_{g} u_{g, d, h}, \quad \forall g \in \mathcal{G}^{\mathrm{C}}, \forall d, \forall h \\
& \underline{P}_{g} u_{g, d, h} \leq p_{g, d, h}^{\mathrm{DA}} \leq \bar{P}_{g} u_{g, d, h}, \quad \forall g \in \mathcal{G}^{\mathrm{E}}, \forall d, \forall h \\
& 0 \leq w_{k, d, h}^{\mathrm{DA}} \leq \bar{W}_{k, d, h}^{\mathrm{DA}} \bar{X}_{k}, \quad \forall k \in \mathcal{K}^{\mathrm{E}}, \forall d, \forall h \\
& 0 \leq w_{k, d, h}^{\mathrm{DA}} \leq \bar{W}_{k, d, h}^{\mathrm{DA}} x_{k}, \quad \forall k \in \mathcal{K}^{\mathrm{C}}, \forall d, \forall h \\
& R_{g}^{-} \leq\left(p_{g, d,(h=1)}^{\mathrm{DA}}-P_{g, d}^{\mathrm{ini}}\right) \leq R_{g}^{+}, \quad \forall g \in \mathcal{G}^{\mathrm{E}}, \forall d
\end{aligned}
$$




$$
\begin{aligned}
& R_{g}^{-} \leq\left(p_{g, d, h}^{\mathrm{DA}}-p_{g, d,(h-1)}^{\mathrm{DA}} \leq R_{g}^{+}, \quad \forall g \in \mathcal{G}^{\mathrm{E}}, \forall d, \forall h>1\right. \\
& \sum_{o} u_{g, o}^{\mathrm{opt}} R_{g}^{\mathrm{opt}-} \leq\left(p_{g, d,(h=1)}^{\mathrm{DA}}-P_{g, d}^{\mathrm{ini}}\right) \leq \sum_{o} u_{g, o}^{\mathrm{opt}} R_{g}^{\mathrm{opt}+}, \quad \forall g \in \mathcal{G}^{\mathrm{C}}, \forall d \\
& \sum_{o} u_{g, o}^{\mathrm{opt}} R_{g}^{\mathrm{opt}-} \leq\left(p_{g, d, h}^{\mathrm{DA}}-p_{g, d,(h-1)}^{\mathrm{DA}}\right) \leq \sum_{o} u_{g, o}^{\mathrm{opt}} R_{g}^{\mathrm{opt}+}, \\
& \quad \forall g \in \mathcal{G}^{\mathrm{C}}, \forall d, \forall h>1 \\
& -\pi \leq \theta_{n, d, h}^{\mathrm{DA}} \leq \pi, \quad \forall n, \forall d, \forall h \\
& \theta_{(n=1), d, h}^{\mathrm{DA}}=0, \quad \forall d, \forall h \\
& \frac{1}{X_{n, m}^{\mathrm{line}}}\left(\theta_{n, d, h}^{\mathrm{DA}}-\theta_{m, d, h}^{\mathrm{DA}} \leq F_{n, m}, \quad \forall n, \forall m \in \Omega_{n}, \forall d, \forall h\right. \\
& c_{g, d,(h=1)}^{\mathrm{SU}} \geq \lambda_{g}^{\mathrm{SU}}\left(u_{g, d,(h=1)}-U_{g, d}^{\mathrm{ini}}\right), \quad \forall g \in \mathcal{G}, \forall d \\
& c_{g, d, h}^{\mathrm{SU}} \geq \lambda_{g}^{\mathrm{SU}}\left(u_{g, d, h}-u_{g, d,(h-1)}\right), \quad \forall g \in \mathcal{G}, \forall d, \forall h>1 \\
& c_{g, d, h}^{\mathrm{SU}} \geq 0, \quad \forall g \in \mathcal{G}, \forall d, \forall h \\
& c_{g, d,(h=1)}^{\mathrm{SD}} \geq \lambda_{g}^{\mathrm{SD}}\left(U_{g, d}^{\mathrm{ini}}-u_{g, d,(h=1)}\right), \quad \forall g \in \mathcal{G}, \forall d \\
& c_{g, d, h}^{\mathrm{SD}} \geq \lambda_{g}^{\mathrm{SD}}\left(u_{g, d,(h-1)}-u_{g, d, h}\right), \quad \forall g \in \mathcal{G}, \forall d, \forall h>1 \\
& c_{g, d, h}^{\mathrm{SD}} \geq 0, \quad \forall g \in \mathcal{G}, \forall d, \forall h .
\end{aligned}
$$

Constraints (3a) represent the power balance in the day-ahead stage at each node, for each representative day, at every hour. Constraints (3b)-(3e) enforce lower and upper bounds for power production of existing and candidate conventional units, as well as existing and candidate wind farms, respectively, for each day and every hour. Constraints (3f) and (3g) limit the ramp rates for existing units, i.e., the change in hourly power production may not exceed the maximum ramp-up and ramp-down rate of each conventional unit, at every day and every hour. Similarly, constraints (3h) and (3i) ensure the ramping limits for candidate units. In particular, constraints (3f) and (3h) enforce the ramping limit in the first hour of each representative day. Constraints $(3 \mathrm{j})$ restrict nodal voltage angles in the scheduling stage, while constraints (3k) set node $n=1$ as the reference node. Constraints (3l) enforce the capacity limit of each transmission line. Constraints (3m)-(3o) and (3p)-(3r) calculate the start-up and shut-down costs of the conventional units, respectively. The explicit modeling of operational reserve requirements to cope with uncertainty from renewable energy generation is not necessary in this setup, since the stochastic UC model optimizes day-ahead decisions with respect to all plausible scenarios in real time $[29,43]$. The second-stage constraints of the proposed GEP model are given by (4) below:

$$
\begin{gathered}
\sum_{g \in \mathcal{G}_{n}}\left(p_{g, d, h}^{\mathrm{DA}}+r_{g, d, h, s}\right)+\sum_{k \in \mathcal{K}_{n}}\left(W_{k, d, h, s}-w_{k, d, h, s}^{\mathrm{SP}}\right)-\sum_{l \in \mathcal{L}_{n}}\left(L_{l, d, h}-p_{l, d, h, s}^{\mathrm{SH}}\right) \\
=\sum_{m \in \Omega_{n}} \frac{1}{X_{n, m}^{\mathrm{line}}}\left(\theta_{n, d, h, s}^{\mathrm{RT}}-\theta_{m, d, h, s}^{\mathrm{RT}}\right), \quad \forall n, \forall d, \forall h, \forall s
\end{gathered}
$$




$$
\begin{aligned}
& 0 \leq p_{l, d, h, s}^{\mathrm{SH}} \leq L_{l, d, h}, \quad \forall l, \forall d, \forall h, \forall s \\
& 0 \leq w_{k, d, h, s}^{\mathrm{SP}} \leq W_{k, d, h, s} \bar{X}_{k}, \quad \forall k \in \mathcal{K}^{\mathrm{E}}, \forall d, \forall h, \forall s \\
& 0 \leq w_{k, d, h, s}^{\mathrm{SP}} \leq W_{k, d, h, s} x_{k}, \quad \forall k \in \mathcal{K}^{\mathrm{C}}, \forall d, \forall h, \forall s \\
& \underline{x}_{g} u_{g, d, h} \leq\left(r_{g, d, h, s}+p_{g, d, h}^{\mathrm{DA}}\right) \leq \bar{x}_{g} u_{g, d, h}, \quad \forall g \in \mathcal{G}^{\mathrm{C}}, \forall d, \forall h, \forall s \\
& \underline{P}_{g} u_{g, d, h} \leq\left(r_{g, d, h, s}+p_{g, d, h}^{\mathrm{DA}}\right) \leq \bar{P}_{g} u_{g, d, h}, \quad \forall g \in \mathcal{G}^{\mathrm{E}}, \forall d, \forall h, \forall s \\
& R_{g}^{-} \leq\left[\left(p_{g, d,(h=1)}^{\mathrm{DA}}+r_{g, d,(h=1), s}\right)-\left(P_{g, d}^{\mathrm{ini}}+R_{g, d}^{\mathrm{ini}}\right)\right] \leq R_{g}^{+}, \\
& \quad \forall g \in \mathcal{G}^{\mathrm{E}}, \forall d, \forall s \\
& R_{g}^{-} \leq\left[\left(p_{g, d, h}^{\mathrm{DA}}+r_{g, d, h, s}\right)-\left(p_{g, d,(h-1)}^{\mathrm{DA}}+r_{g, d,(h-1), s}\right)\right] \leq R_{g}^{+}, \\
& \quad \forall g \in \mathcal{G}^{\mathrm{E}}, \forall d, \forall h>1, \forall s \\
& \sum_{o} u_{g, o}^{\mathrm{opt}} R_{g}^{\mathrm{opt}-} \leq\left[\left(p_{g, d,(h=1)}^{\mathrm{DA}}+r_{g, d,(h=1), s}\right)-\left(P_{g, d}^{\mathrm{ini}}+R_{g, d}^{\mathrm{ini}}\right)\right] \leq \sum_{o} u_{g, o}^{\mathrm{opt}} R_{g}^{\mathrm{opt}+} \\
& \quad \forall g \in \mathcal{G}^{\mathrm{C}}, \forall d, \forall s \\
& \sum_{o} u_{g, o}^{\mathrm{opt}} R_{g}^{\mathrm{opt}-} \leq\left[\left(p_{g, d, h}^{\mathrm{DA}}+r_{g, d, h, s}\right)-\left(p_{g, d,(h-1)}^{\mathrm{DA}}+r_{g, d,(h-1), s}\right)\right] \leq \sum_{o} u_{g, o}^{\mathrm{opt}} R_{g}^{\mathrm{opt}+}, \\
& \quad \forall g \in \mathcal{G}^{\mathrm{C}}, \forall d, \forall h>1, \forall s \\
& \quad-\pi \leq \theta_{n, d, h, s}^{\mathrm{RT}} \leq \pi, \quad \forall n, \forall d, \forall h, \forall s \\
& \theta_{(n=1), d, h, s}^{\mathrm{RT}}=0, \quad \forall d, \forall h, \forall s \\
& \frac{1}{X_{n, m}^{\mathrm{Iine}}}\left(\theta_{n, d, h, s}^{\mathrm{RT}}-\theta_{m, d, h, s}^{\mathrm{RT}} \leq F_{n, m}, \quad \forall n, \forall m \in \Omega_{n}, \forall d, \forall h, \forall s .\right.
\end{aligned}
$$

Constraints (4a) enforce the power balance in real-time operation at each node and for each representative day at every hour under each scenario, resolving the deviations of actual wind production with regulating power provision by conventional units, and/or wind power curtailment, and/or load shedding. The uncertain parameter $W_{k, d, h, s}$ in (4a) characterizes the wind power uncertainty. Constraints (4b)-(4d) restrict the amount of curtailed load as well as curtailed wind power by existing and candidate farms, respectively. The wind power spillage is a balancing action, which occurs only if there is no generation unit whose production level can be reduced. One can enforce a higher use of wind power by adding wind spillage cost (it is zero in the current setup) or adding a constraint for renewable portfolio standard in terms of energy and not power. However, both may increase the system cost. For each existing and candidate conventional unit, respectively, constraints (4e) and (4f) enforce lower and upper bounds for its actual power production, i.e., tentative production schedule at the first stage plus its regulating power provided in the real-time stage. Constraints $(4 \mathrm{~g})-(4 \mathrm{j})$ enforce ramping limits for actual production of existing and candidate conventional units. Constraints (4k) enforce the lower and upper bounds of the real-time nodal voltage angles, while (4l) sets node $n=1$ as the reference bus. Constraints $(4 \mathrm{~m})$ limit the capacity of each transmission line in real-time under all scenarios. Note that equations (4a) and (4e)-(4j) link the first and second stages. Note also that the current form of GEP model is mixed-integer and non-linear. The sources of non-linearity are 
constraints (3b) and (4e) as they include the product of binary and continuous variables, i.e., $\underline{x}_{g} u_{g, d, h}$ and $\bar{x}_{g} u_{g, d, h}$. Pursuing linearity, constraints (3b) and (4e) are linearized in an exact way using two auxiliary continuous variables $\underline{\Phi}_{g, d, h}$ and $\bar{\Phi}_{g, d, h}$ as well as a large positive constant $M$, e.g., maximum capacity of expansion options. For this linearization, the following constraints are included in the GEP problem:

$$
\begin{aligned}
& -u_{g, d, h} M \leq \underline{\Phi}_{g, d, h} \leq u_{g, d, h} M, \quad \forall g \in \mathcal{G}^{\mathrm{C}}, \forall d, \forall h \\
& -\left(1-u_{g, d, h}\right) M \leq\left(\underline{\Phi}_{g, d, h}-\underline{x}_{g}\right) \leq\left(1-u_{g, d, h}\right) M, \quad \forall g \in \mathcal{G}^{\mathrm{C}}, \forall d, \forall h \\
& -u_{g, d, h} M \leq \bar{\Phi}_{g, d, h} \leq u_{g, d, h} M, \quad \forall g \in \mathcal{G}^{\mathrm{C}}, \forall d, \forall h \\
& -\left(1-u_{g, d, h}\right) M \leq\left(\bar{\Phi}_{g, d, h}-\bar{x}_{g}\right) \leq\left(1-u_{g, d, h}\right) M, \quad \forall g \in \mathcal{G}^{\mathrm{C}}, \forall d, \forall h .
\end{aligned}
$$

The functioning of constraints (5) are as follows: if the optimal value of binary variable $u_{g, d, h}$ is zero, then (5c) enforces $\bar{\Phi}_{g, d, h}=0$, while (5d) is inactive. Otherwise, if the optimal value of binary variable $u_{g, d, h}=1$, then (5c) is inactive, while (5d) enforces $\bar{\Phi}_{g, d, h}=\bar{x}_{g}$. Similarly, constraints (5a) and (5b) set the optimal values for $\underline{\Phi}_{g, d, h}$ and $\underline{x}_{g}$. Therefore, the non-linear equations (3b) and (4e) in the GEP problem can now be substituted by the following two linear equations:

$$
\begin{aligned}
& \underline{\Phi}_{g, d, h} \leq p_{g, d, h}^{\mathrm{DA}} \leq \bar{\Phi}_{g, d, h}, \quad \forall g \in \mathcal{G}^{\mathrm{C}}, \forall d, \forall h \\
& \underline{\Phi}_{g, d, h} \leq\left(r_{g, d, h, s}+p_{g, d, h}^{\mathrm{DA}}\right) \leq \bar{\Phi}_{g, d, h}, \quad \forall g \in \mathcal{G}^{\mathrm{C}}, \forall d, \forall h, \forall s .
\end{aligned}
$$

\section{Scalability strategy: multi-cut Benders' decomposition}

The proposed mixed-integer linear GEP model (1)-(5) potentially becomes computationally intractable if many representative days and scenarios are considered. For scalability purposes, this section develops a solution strategy based on a multi-cut Benders' decomposition algorithm [5,6] to solve problem (1)-(5). Decomposition means the splitting up of a mathematical programming problem into a set of separate smaller problems that are each easier to solve compared to the original problem and then reintegrate them in an iterative manner to get an overall solution [10]. Benders' decomposition procedure has been extensively applied to stochastic two-stage programming problems, known also as the L-shaped method $[10,55,65]$.

\subsection{Decomposition by scenario and representative day}

Fixing the first-stage (i.e., expansion and day-ahead UC) variables to given values in the original problem (1)-(5) decomposes the proposed problem into a set of continuous linear subproblems, one per representative day and scenario, each representing the real-time operation in the second stage. These fixing variables are referred to as complicating variables [10] and include $x_{k}, p_{g, d, h}^{\mathrm{DA}}, u_{g, d, h}, \Phi_{g, d, h}, \bar{\Phi}_{g, d, h}$, and ramp $p_{g}$. Note that dummy variable $\operatorname{ramp}_{g}$ is defined here as $\sum_{o} u_{g, o}^{\mathrm{opt}} R_{g, o}^{\mathrm{opt}+} \forall g \in \mathcal{G}^{C}$. We then formulate a MILP, the so-called master problem, to update the value of complicat- 
ing variables in each iteration. A requirement for Benders' decomposition algorithm to work is convexity of the original problem with respect to complicating variables [10]. Since the original problem (1)-(5) does not necessarily have a convex solution space, Benders' decomposition is not generally applicable to this problem. The use of Benders' decomposition with either uni- or multi-cut algorithm becomes a heuristic. However, with an increasing number of scenarios, the objective function of the original problem as the expected value over all scenarios asymptotically convexifies [34], and thereby Benders' convergence might be obtained. Increasing the number of scenarios smoothens the objective function. The reason for this is that with increasing number of scenarios, the probability of each scenario lowers, and thus, the weight for the scenario in the objective function reduces, provided that the data for added scenarios is not too volatile. Briefly, the proposed problem is sufficiently convexified by considering a large enough number of scenarios. The numerical analyses in Sect. 5.4 verify the well-functioning of the proposed Benders' algorithm.

\subsection{Subproblems}

For given values for complicating variables, i.e., $x_{k}^{\text {fixed(i) }}, p_{g, d, h}^{\text {DA, fixed(i) }}, u_{g, d, h}^{\text {fixed(i) }}, \Phi_{g, d, h}^{\text {,fixed(i) }}$, $\bar{\Phi}_{g, d, h}^{\text {,fixed(i) }}$, and ramp $_{g}^{\text {fixed(i) }}$, the continuous linear subproblem associated with representative day $d$ and scenario $s$ is formulated as (6) below. The optimization variables, all pertaining to Benders' iteration $i$, for each subproblem (6) are the elements of the set $\Xi_{d, s}^{\mathrm{SP}(\mathrm{i})}=\left\{z_{d, s}^{(\mathrm{i})}, x_{k}^{(\mathrm{i})}, p_{g, d, h}^{\mathrm{DA}(\mathrm{i})}, u_{g, d, h}^{(i)}, \underline{\Phi}_{g, d, h}^{(\mathrm{i})}, \bar{\Phi}_{g, d, h}^{(\mathrm{i})}, \operatorname{ramp}_{g}^{(\mathrm{i})} r_{g, d, h, s}^{(\mathrm{i})}, w_{k, d, h, s}^{\mathrm{SP}(\mathrm{i})}, p_{l, d, h, s}^{\mathrm{SH}(\mathrm{i})}\right.$, $\left.\theta_{n, d, h, s}^{\mathrm{RT}(\mathrm{i})}\right\}$.

$$
\left\{\underset{\Xi_{d, s}^{\mathrm{SP}(\mathrm{i})}}{\operatorname{Minimize}} z_{d, s}^{(\mathrm{i})}=\sum_{g \in \mathcal{G}, h} C_{g} r_{g, d, h, s}^{(\mathrm{i})}+\sum_{l \in \mathcal{L}, h} V_{l}^{\mathrm{SH}} p_{l, d, h, s}^{\mathrm{SH}(\mathrm{i})}\right.
$$

subject to:

$$
\begin{aligned}
& \text { (4a) }-(4 d) \\
& (4 \mathrm{f})-(4 \mathrm{~h}) \\
& \operatorname{ramp}_{g} \leq\left[\left(p_{g, d,(h=1)}^{\mathrm{DA}}+r_{g, d,(h=1), s}\right)-\left(P_{g, d}^{\mathrm{ini}}+R_{g, d}^{\mathrm{ini}}\right)\right] \leq \operatorname{ramp}_{g}, \\
& \forall g \in \mathcal{G}^{\mathrm{C}} \\
& \operatorname{ramp}_{g} \leq\left[\left(p_{g, d, h}^{\mathrm{DA}}+r_{g, d, h, s}\right)-\left(p_{g, d,(h-1)}^{\mathrm{DA}}+r_{g, d,(h-1), s}\right)\right] \leq \operatorname{ramp}_{g}, \\
& \forall g \in \mathcal{G}^{\mathrm{C}}, \forall h>1
\end{aligned}
$$

$$
\begin{array}{ll}
x_{k}^{(i)}=x_{k}^{\text {fixed(i) }} & : \lambda_{k, d, s}^{x^{(\mathrm{i})}}, \quad \forall k \in \mathcal{K}^{C} \\
p_{g, d, h}^{\mathrm{DA}(\mathrm{i})}=p_{g, d, h}^{\mathrm{DA}, \mathrm{fixed}(\mathrm{i})} & : \lambda_{g, d, h, s}^{p^{\mathrm{DA}(\mathrm{i})}}, \quad \forall g, \forall h
\end{array}
$$




$$
\begin{array}{ll}
u_{g, d, h}^{(\mathrm{i})}=u_{g, d, h}^{\text {fixed(i) }} & : \lambda_{g, d, h, s}^{u(\mathrm{i})}, \quad \forall g, \forall h \\
\underline{\Phi}_{g, d, h}^{(\mathrm{i})}=\underline{\Phi}_{g, d, h}^{\text {fixed(i) }} & : \lambda \frac{\Phi}{g, d, h, s}^{(\mathrm{i})}, \quad \forall g \in \mathcal{G}^{C}, \forall h \\
\bar{\Phi}_{g, d, h}^{(\mathrm{i})}=\bar{\Phi}_{g, d, h}^{\text {fixed(i) }} & : \lambda_{g, d, h, s}^{\bar{\Phi}^{(\mathrm{i})}}, \quad \forall g \in \mathcal{G}^{C}, \forall h \\
r a m p_{g}^{(i)}=r a m p_{g}^{\text {fixed(i) }}: \lambda_{g, d, s}^{\text {ramp }^{(\mathrm{i})}}, \quad \forall g \in \mathcal{G}^{C}
\end{array}
$$

\}$\forall d, \forall s$

Objective function (6a) minimizes the imbalance cost of the system in representative day $d$ incurred in the real-time operation under scenario $s$. Constraint (6b)-(6g) includes all second-stage constraints, while (6h)-(6m) fix the complicating variables to predefined values. The dual variables of fixing constraints $(6 \mathrm{~h})-(6 \mathrm{~m})$, i.e., $\lambda_{k, d, s}^{x^{(\mathrm{i})}}$, $\lambda_{g, d, h, s}^{p^{\mathrm{DA}(\mathrm{i})}}, \lambda_{g, d, h, s}^{u(\mathrm{i})}, \lambda \frac{\Phi}{g, d, h, s}^{(\mathrm{i})}, \lambda_{g, d, h, s}^{\Phi^{(\mathrm{i})}}, \lambda_{g, d, s}^{\mathrm{ramp}^{(\mathrm{i})}}$, provide sensitivities required for building Benders' optimality cuts in the master problem, which will be explained later in ( $8 \mathrm{~b})$. For given values for complicating variables, the solution of the subproblem above provides optimal values for the non-complicating (i.e., second-stage) variables and the dual variable vector associated with fixing those constraints. It is worth mentioning that in the subproblems, $u_{g, d, h}^{(\mathrm{i})}$ is treated as a continuous variable, although its value is fixed to a binary parameter ( 0 or 1$)$. Therefore, each subproblem is continuous and linear. The solution of all subproblems, one per representative day and scenario, allows computing an upper bound for the Benders' algorithm at iteration $i$ as follows:

$$
\begin{aligned}
z_{u p}^{(\mathrm{i})}= & \sum_{g \in \mathcal{G}^{C}} I_{g} \bar{x}_{g}^{\mathrm{fixed}(\mathrm{i})}+\sum_{k \in \mathcal{K}^{C}} I_{k} x_{k}^{\mathrm{fixed}(\mathrm{i})} \\
& +\sum_{g, d, h} \sigma_{d}\left(c_{g, d, h}^{\mathrm{SU}, \text { fixed(i) }}+c_{g, d, h}^{\mathrm{SD}, \text { fixed(i) }}+C_{g} p_{g, d, h}^{\mathrm{DA}, \text { fixed(i) }}\right)+z^{(\mathrm{i})} .
\end{aligned}
$$

The values of $c_{g, d, h}^{\mathrm{SU}, \text { fixed(i) }}$ and $c_{g, d, h}^{\mathrm{SD}, \text { fixed(i) }}$ are calculated using fixed values for $u_{g, d, h}^{\text {fixed(i), }}$ while $z^{(\mathrm{i})}$ is calculated as $\sum_{d, s} \rho_{s} \sigma_{d} z_{d, s}^{(\mathrm{i})}$.

\subsection{Master problem}

The master problem is formulated as mixed-integer linear problem (8) below. All variables refer to Benders' iteration $i$.

$$
\begin{aligned}
\underset{\Xi^{\mathrm{MP}(\mathrm{i})}}{\operatorname{Minimize}} z_{d o w n}^{(\mathrm{i})}= & \sum_{g \in \mathcal{G}^{C}} I_{g} \bar{x}_{g}^{(\mathrm{i})}+\sum_{k \in \mathcal{K}^{C}} I_{k} x_{k}^{(\mathrm{i})} \\
& +\sum_{g, d, h} \sigma_{d}\left(c_{g, d, h}^{\mathrm{SU}(\mathrm{i})}+c_{g, d, h}^{\mathrm{SD}(\mathrm{i})}+C_{g} p_{g, d, h}^{\mathrm{DA}(\mathrm{i})}\right)+\sum_{s} \rho_{s} \alpha_{s}^{(\mathrm{i})}
\end{aligned}
$$

subject to: 


$$
\begin{aligned}
& \sum_{d} \sigma_{d} z_{d, s}^{(\mathrm{j})}+\sum_{k \in \mathcal{K}^{C}, d} \sigma_{d} \lambda_{k, d, s}^{x^{(\mathrm{j})}}\left(x_{k}^{(\mathrm{i})}-x_{k}^{(\mathrm{j})}\right) \\
& +\sum_{g, d, h} \sigma_{d} \lambda_{g, d, h, s}^{p^{\mathrm{DA}(\mathrm{j})}}\left(p_{g, d, h}^{\mathrm{DA}(\mathrm{i})}-p_{g, d, h}^{\mathrm{DA}(\mathrm{j})}\right) \\
& +\sum_{g, d, h} \sigma_{d} \lambda_{g, d, h, s}^{u^{(\mathrm{j})}}\left(u_{g, d, h}^{(\mathrm{i})}-u_{g, d, h}^{(\mathrm{j})}\right) \\
& +\sum_{g \in \mathcal{G}^{C}, d, h} \sigma_{d} \lambda \frac{\Phi}{g, d, h, s}^{(\mathrm{j})}\left(\underline{\Phi}_{g, d, h}^{(\mathrm{i})}-\underline{\Phi}_{g, d, h}^{(\mathrm{j})}\right) \\
& +\sum_{g \in \mathcal{G}^{C}, d, h} \sigma_{d} \lambda_{g, d, h, s}^{\bar{\Phi}^{(\mathrm{j})}}\left(\bar{\Phi}_{g, d, h}^{(\mathrm{i})}-\bar{\Phi}_{g, d, h}^{(\mathrm{j})}\right) \\
& +\sum_{g \in \mathcal{G}^{C}, d} \sigma_{d} \lambda_{g, d, s}^{r a m p}\left(\operatorname{ramp}_{g}^{(\mathrm{i})}-\operatorname{ramp}_{g}^{(\mathrm{j})}\right) \leq \alpha_{s}^{(\mathrm{i})} \text {, } \\
& \forall s, \forall j=1, \ldots, i-1 \\
& \alpha_{s}^{(i)} \geq \alpha^{\text {down }}, \quad \forall s \\
& \text { (2a) }-(2 \mathrm{f}) \\
& \text { (3a), }(3 \mathrm{c})-(3 \mathrm{r}) \\
& (5 \mathrm{a})-(5 \mathrm{e}) \\
& u_{g, d, h}^{(\mathrm{i})} \in\{0,1\} \\
& \underline{P}_{g} u_{g, d, h}^{(\mathrm{i})} \leq p_{g, d, h}^{\mathrm{DA}(\mathrm{i})}+\left[\min _{s} r_{g, d, h, s}^{(\mathrm{i}-1)}\right] u_{g, d, h}^{(\mathrm{i})}, \quad \forall g \in \mathcal{G}^{E}, \forall d, \forall h \\
& p_{g, d, h}^{\mathrm{DA}(\mathrm{i})}+\left[\max _{s} r_{g, d, h, s}^{(\mathrm{i}-1)}\right] u_{g, d, h}^{(\mathrm{i})} \leq \bar{P}_{g} u_{g, d, h}^{(i)}, \quad \forall g \in \mathcal{G}^{E}, \forall d, \forall h \\
& \underline{\Phi}_{g, d, h}^{(\mathrm{i})} \leq p_{g, d, h}^{\mathrm{DA}(\mathrm{i})}+\left[\min _{s} r_{g, d, h, s}^{(\mathrm{i}-1)}\right] u_{g, d, h}^{(\mathrm{i})}, \quad \forall g \in \mathcal{G}^{C}, \forall d, \forall h \\
& p_{g, d, h}^{\mathrm{DA}(\mathrm{i})}+\left[\max _{s} r_{g, d, h, s}^{(\mathrm{i}-1)}\right] u_{g, d, h}^{(\mathrm{i})} \leq \bar{\Phi}_{g, d, h}^{(\mathrm{i})}, \quad \forall g \in \mathcal{G}^{C}, \forall d, \forall h
\end{aligned}
$$

The objective function (8a) corresponds to (1), where the auxiliary variable $\alpha_{s}^{(i)}$ represents the real-time operation cost under scenario $s$. The optimization variables of master problem (8) are the elements of the set $\Xi^{\mathrm{MP}(\mathrm{i})}=\left\{z_{d o w n}^{(\mathrm{i})}, x_{k}^{(\mathrm{i})}, \bar{x}_{g}^{(\mathrm{i})}, \underline{x}_{g}^{(\mathrm{i})}, u_{g}^{\text {opt(i) }}\right.$, $\left.\Phi_{g, d, h}^{(\mathrm{i})}, \bar{\Phi}_{g, d, h}^{(\mathrm{i})}, \theta_{n, d, h}^{\mathrm{DA}(\mathrm{i})}, c_{g, d, h}^{\mathrm{SU}(\mathrm{i})}, c_{g, d, h}^{\mathrm{SD}(\mathrm{i})}, p_{g, d, h}^{\mathrm{DA}(\mathrm{i})}, w_{k, d, h}^{\mathrm{DA}(\mathrm{i})}, u_{g, d, h}^{(\mathrm{i})}, \operatorname{ramp}_{g}^{(\mathrm{i})}, \alpha_{s}^{(\mathrm{i})}\right\}$. The optimal objective function value of the master problem provides a lower bound for the optimal objective function value of the original problem, because problem (8) is a relaxation of the original problem. The solution of this master problem provides the updated values for the complicating variables that are fixed within the subproblems. Constraints (8b), one per scenario, are Benders' optimality cuts. These constraints tighten the feasible 
space of the master problem over iterations using the values obtained for dual variable vector $\lambda$ in the subproblems. In a regular unicut Benders' decomposition implementation, a single cut is generated considering all scenarios [5]; however, a multi-cut version of Benders' decomposition generates one cut per scenario [6]. The multi-cut Benders' decomposition generally converges faster than the regular one, but at the cost of increased dimension for the master problem especially in problems with a high number of scenarios $[33,63]$. Note that the optimality cuts $(8 \mathrm{~b})$ are sufficient, i.e., Benders' feasibility cuts are not required in this implementation since the subproblems (6) are always feasible for any given values of the complicating variables due to the possibility of load shedding and wind power spillage. In general, the multi-cut algorithm tends to require fewer iterations than the unicut algorithm. However, the trade-offs in terms of computational time are problem-dependent $[57,62,64]$. An alternative approach to the multi-cut version is an adaptive strategy, i.e., starting with the multi-cut version of Benders' algorithm and then switching to the single-cut version after a few iterations $[53,55,65]$, which is outside the scope of this work.

Constraints (8c) impose a lower bound on $\alpha_{s}$ to avoid the master problem to be unbounded in the first iteration. Constraint (8d) includes the generation expansion constraints in the original GEP model, while (8e) consists of the first-stage constraints. In addition, (8f) contains the linearization constraints. Constraints $(8 \mathrm{~h})-(8 \mathrm{k})$ replace the first-stage constraints (3b) and (3c) in order to accelerate the convergence of Benders' decomposition by improving the information flow between subproblems and master problem, as originally proposed in [34]. Note that $\left[\min _{s} r_{g, d, h, s}^{(\mathrm{i}-1)}\right]$ and $\left[\max _{s} r_{g, d, h, s}^{(\mathrm{i}-1)}\right]$ are both parameters obtained from the solution of the subproblems in the previous iteration, and illustrate the minimum and maximum regulating power provided by generation unit $g$ over scenarios $s$ in hour $h$ of representative day $d$. In this way, in addition to Benders' cuts (8b), constraints (8h)-(8k) provide extra link between master problem and subproblems without drastically increasing the computational effort.

\subsection{Benders' algorithm}

The proposed Benders' algorithm works as follows: within a stochastic minimization problem, upper bounds are obtained by fixing the first-stage variables and optimizing the second-stage decisions (subproblems) for each representative day and scenario. Lower bounds are obtained by solving a master problem in the space of the firststage variables, which incorporates lower bounds on the expected second-stage costs. Convergence of the algorithm is achieved by improving the lower bound with the master problem which adds successively dual information of the second-stage costs obtained from the upper-bounding subproblems, which in turn potentially leads to first-stage decisions that improve the upper bound. Lower bounds on the second-stage costs are added to the master problem in the form of optimality cuts determined by the dual multipliers of the subproblems, see Fig. 2.

In turn, these subproblems then provide new information to formulate a more accurate master problem that provides new values of complicating variables. The procedure continues until upper and lower bounds for the optimal value of the objective function converge [10]. The steps for Benders' algorithm are as follows: 


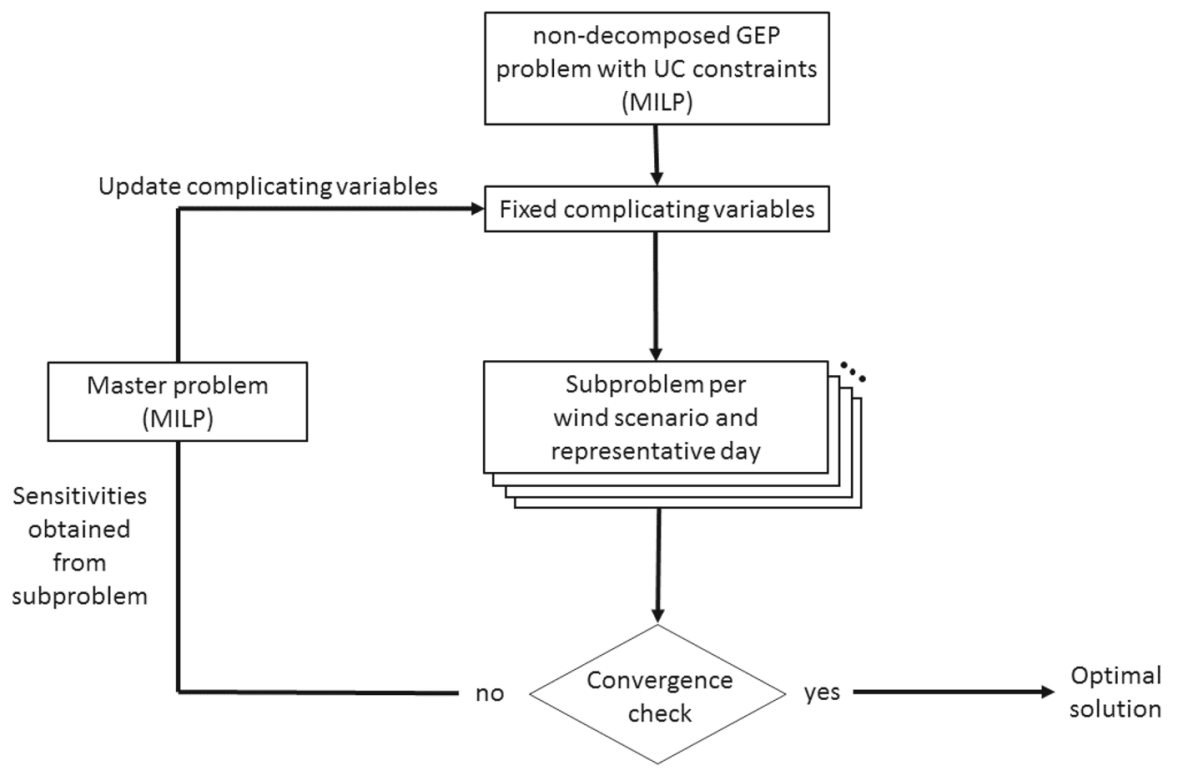

Fig. 2 Flowchart of Benders' Algorithm

1. Input: a small tolerance $\varepsilon$ to control convergence, and initial guesses of the complicating variables, $x_{k}^{(0)}, \Phi_{g, d, h}^{(0)}, \bar{\Phi}_{g, d, h}^{(0)}, p_{g, d, h}^{\mathrm{DA}(0)}, u_{g, d, h}^{(0)}$, and ramp $p_{g}^{(0)}$.

2. Initialization: Set $i=1, z_{\text {down }}^{(\mathrm{i})}=-\infty, x_{k}^{\text {fixed }}=x_{k}^{(0)}, \underline{\Phi}_{g, d, h}^{\text {fixed }}=\underline{\Phi}_{g, d, h}^{(0)}, \bar{\Phi}_{g, d, h}^{\text {fixed }}=$ $\bar{\Phi}_{g, d, h}^{(0)}, p_{g, d, h}^{\mathrm{DA}, \mathrm{fixed}}=p_{g, d, h}^{\mathrm{DA}(0)}, u_{g, d, h}^{\text {fixed }}=u_{g, d, h}^{(0)}$ and $\operatorname{ramp}_{g}^{\mathrm{fixed}}=\operatorname{ramp}_{g}^{(0)}$.

3. Initial representative day: Consider representative day $d=1$.

4. Initial scenario: Consider scenario $s=1$.

5. Subproblem solution: Solve (6) for representative day $d$ and scenario $s$ and calculate $z_{d, s}^{(i)}$.

6. Next scenario: Consider the next scenario, and repeat step 5. If all scenarios have been considered, go to the next step.

7. Next representative day: Consider the next representative day, and repeat steps 4 to 6 . If all representative days have been considered, go to the next step.

8. Convergency check: If $\left|z_{u p}^{(i)}-z_{\text {down }}^{(\mathrm{i})}\right| \leq \varepsilon$, the optimal solution with a level of accuracy $\varepsilon$ has been obtained. Otherwise, calculate $z^{(i)}$ and the sensitivities to build the next Benders' cuts. Then, set $i \longleftarrow i+1$.

9. Master problem solution: Solve (8), calculate $z_{\text {down }}^{(\mathrm{i})}$ and update the values of complicating variables. Then, continue in step 3.

\section{Numerical study}

This section presents numerical results for two case studies. The first illustrative example is based on a six-node power system, whose typology is shown in Fig. 3. This case 


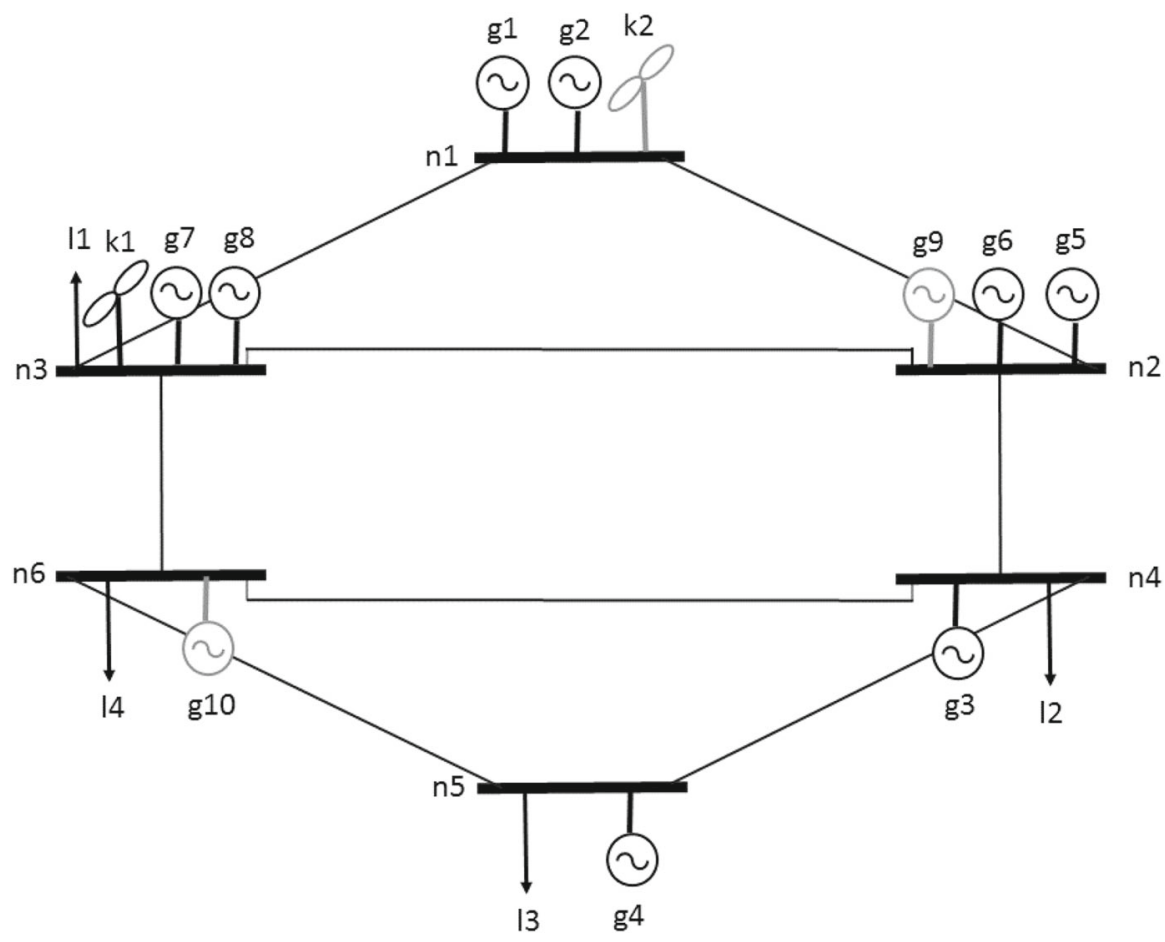

Fig. 3 Topology of the six-node power system

study is in fact a modified version of the system used in [24]. Next, a larger case study is presented based on an updated version of the IEEE 24-node reliability test system [37].

\subsection{Six-node test system: input data}

We use the conventional generation data from [23,24], as well as the load forecast of a regional transmission operator in the PJM market in 2016 [3] and the wind realization data of the Belgian transmission system operator, ELIA [1]. The technical input data for existing and candidate conventional generation units are given in Table 2. Note that conventional generation units $g 1$ to $g 8$ are existing units, while $g 9$ and $g 10$ are the two candidate conventional units to be built, each with five expansion options. In addition to conventional units, an existing wind farm $k 1$ with an installed capacity of $500 \mathrm{MW}$ is located at node $n 3$, while $k 2$ is a candidate wind farm at node $n 1$.

The annualized investment cost for additional wind capacity is $€ 100,000 / \mathrm{MW}$, while it is $€ 75,000 / \mathrm{MW}$ and $€ 20,000 / \mathrm{MW}$ for the candidate generation units $g 9$ and $g 10$, respectively. The capacity of transmission lines $n 3$ to $n 6$ and $n 2$ to $n 4$ is 500 $\mathrm{MW}$, while it is $450 \mathrm{MW}$ for the rest of lines. The reactance of all lines is $0.001 \mathrm{Ohm}$. The renewable portfolio standard factor is set to 0.3 , enforcing the share of renewable capacity to be at least $30 \%$ of the total generation portfolio including all existing and 


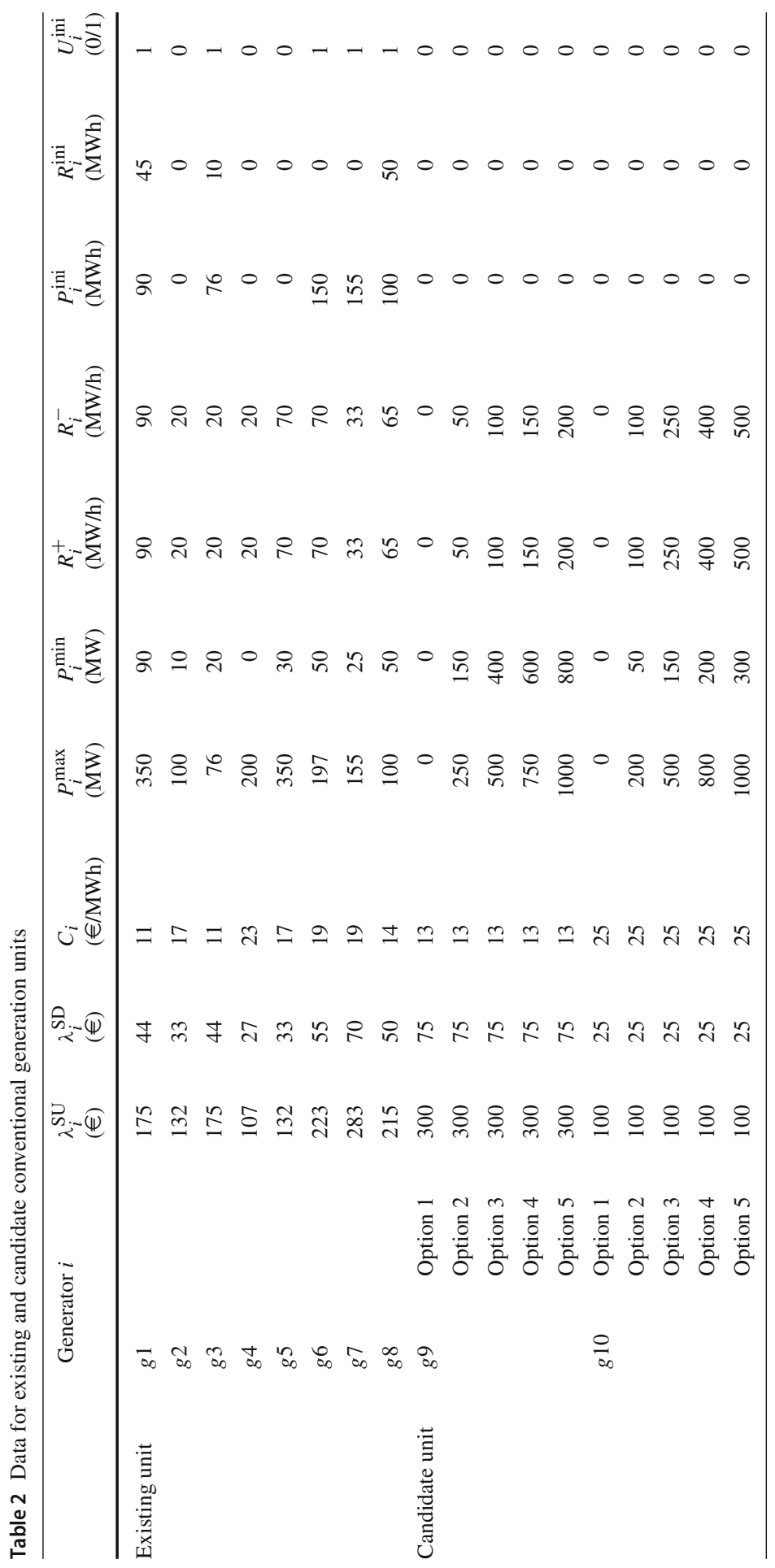




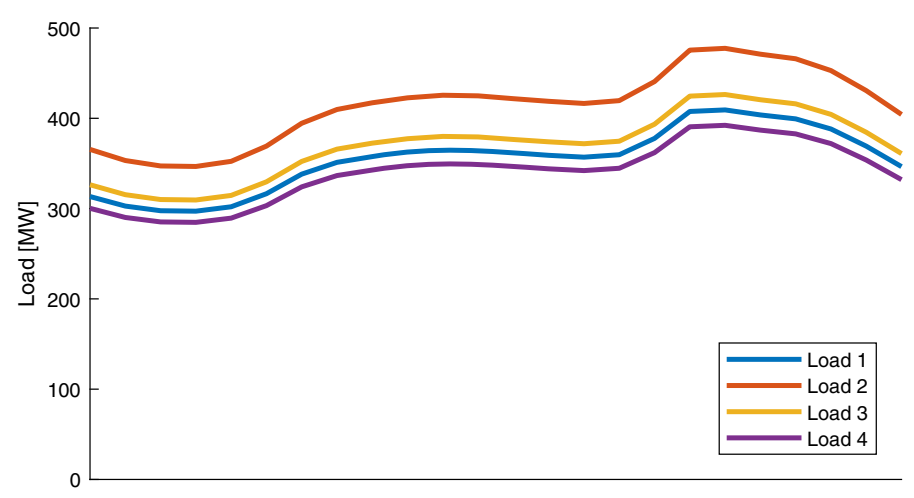

Fig. 4 Daily load profile of demands in a sample representative day

candidate conventional and renewable units. The supply security factor is set to $120 \%$ of maximum load.

We consider ten representative days for modeling the target year, whose weights are equal, i.e., 36.5. This case study includes four demands, and their load profiles over one sample representative day are illustrated in Fig. 4. The value of lost load of each demand is identical, i.e., $€ 300 / \mathrm{MWh}$. In addition, we consider 100 equiprobable wind scenarios. Note that each wind scenario includes wind power realization of each farm (either existing or candidate) over all hours and representative days. In other words, each scenario includes 10 daily wind power profiles (one per representative day) for each farm, as depicted in Fig. 5 for the existing wind farm for one sample representative day. The whole input data and the codes used are available in the electronic companion of the paper [50].

\subsection{Six-node test system: numerical results}

Figure 6 illustrates the evolution of multi-cut Benders' algorithm over iterations. This algorithm converges in the seventh iteration, in which the difference between upper and lower curves goes below the predefined tolerance.

The optimal expansion plan for the six-node system with 100 scenarios incurs expected cost of $€ 206,357,882$. According to the optimal GEP results obtained, a new wind farm with the capacity of 497.7 MW as well as a 800-MW conventional unit are installed. This new conventional unit is the one located at node $n 6$ (i.e., g10) with the minimum production level of $200 \mathrm{MW}$ and ramp rates of $400 \mathrm{MW} / \mathrm{h}$.

We now evaluate the impact of UC constraints on GEP decisions by adding or removing each constraint one at a time. Accordingly, eight cases are defined incorporating different combinations of UC constraints. Starting with the complete original model, different constraints are individually relaxed. These cases, summarized in Table 3, are:

- Case 1: This case refers to a GEP problem with all UC constraints included, i.e., start-up and shut-down costs, minimum production and hourly ramp rate limits of conventional generation units. 


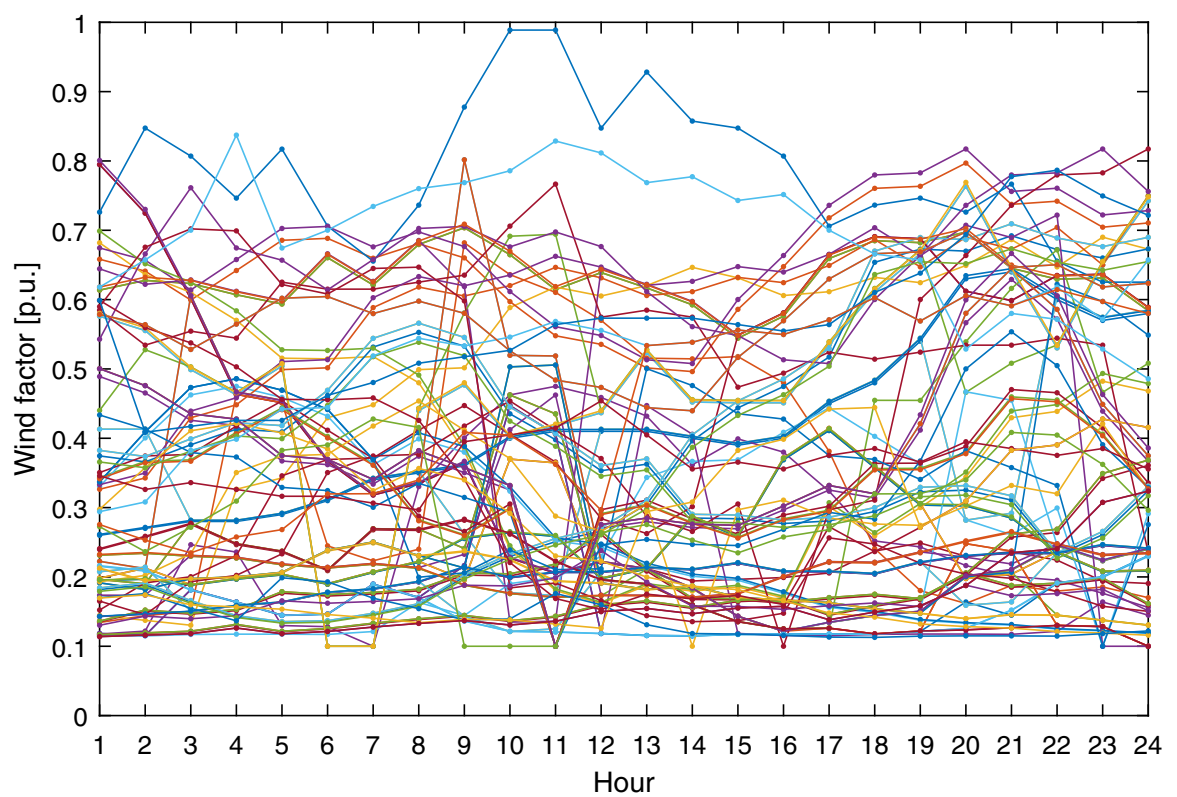

Fig. 5 Per-unit wind realization factor for existing wind farm $k 1$ in a sample representative day under each of 100 scenarios considered

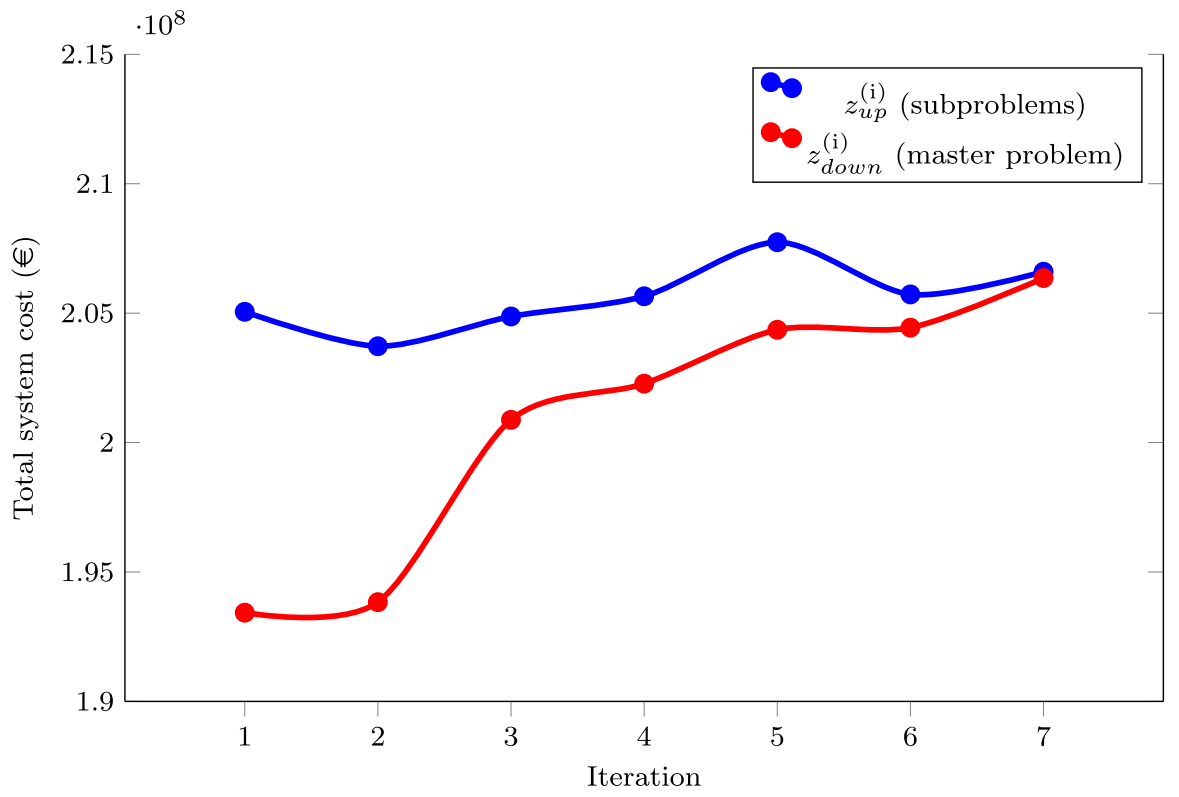

Fig. 6 Six-node test system: evolution of multi-cut Benders' algorithm over iterations. This case considers 10 representative days and 100 scenarios (i.e., 1000 subproblems) 
Table 3 Summary of cases

\begin{tabular}{llll}
\hline Case & $\begin{array}{l}\text { Start-up and shut-down } \\
\text { costs of units included? }\end{array}$ & $\begin{array}{l}\text { Minimum production } \\
\text { level of units included? }\end{array}$ & $\begin{array}{l}\text { Ramp limits of units } \\
\text { included? }\end{array}$ \\
\hline Case 1 & Yes & Yes & Yes \\
Case 2 & No & Yes & Yes \\
Case 3 & Yes & No & Yes \\
Case 4 & Yes & Yes & No \\
Case 5 & No & No & No \\
Case 6 & Yes & No & No \\
Case 7 & No & Yes & No \\
Case 8 & No & No & Yes \\
\hline
\end{tabular}

- Case 2: This case is similar to Case 1, but the start-up and shut-down costs of conventional units is excluded.

- Case 3: This case is similar to Case 1, but the minimum production level of generation units is fixed to zero.

- Case 4: This case is similar to Case 1, but the hourly ramp rate limits of generation units are excluded.

- Case 5: This case refers to a GEP problem with economic dispatch constraints only; none of the UC constraints is included.

- Case 6: This case is similar to Case 5, but the start-up and shut-down costs of conventional units is included.

- Case 7: This case is similar to Case 5, but the actual non-zero minimum production level of generation units is included.

- Case 8: This case is similar to Case 5, but the hourly ramp rate limits of generation units are included.

Figure 7 summarizes the results obtained from Cases 1 to 8 . The capacity of newly built conventional and wind units as well as the total expected system cost, i.e., the optimal value of objective function (1), vary depending on the relaxation or enforcement of UC constraints. We consider Case 1 as a benchmark since it enforces all UC constraints. Among Cases 2 to 4, the relaxation of ramping constraints (Case 4) causes the largest underestimation of system cost and new capacity required, while the other relaxations (Cases 2 and 3) yield a slighter underestimation. In particular, Case 2 excluding the start-up and shut-down costs of conventional units obtains the same expansion decisions compared to those in Case 1, but a lower system cost. Furthermore, Fig. 7 shows that the incorporation of hourly ramping constraints into the GEP model (Case 8) significantly reduces the cost error, and in this specific example, it even leads to the correct investment decision (i.e., the same expansion decisions as in Case 1). The other constraints in this specific example seem to be considerably less effective. For example, incorporating the start-up and shut-down costs of units (Case 6) or minimum production level of units (Case 7) while ignoring ramping constraints still causes wrong investment decisions with a comparatively high system cost under- 


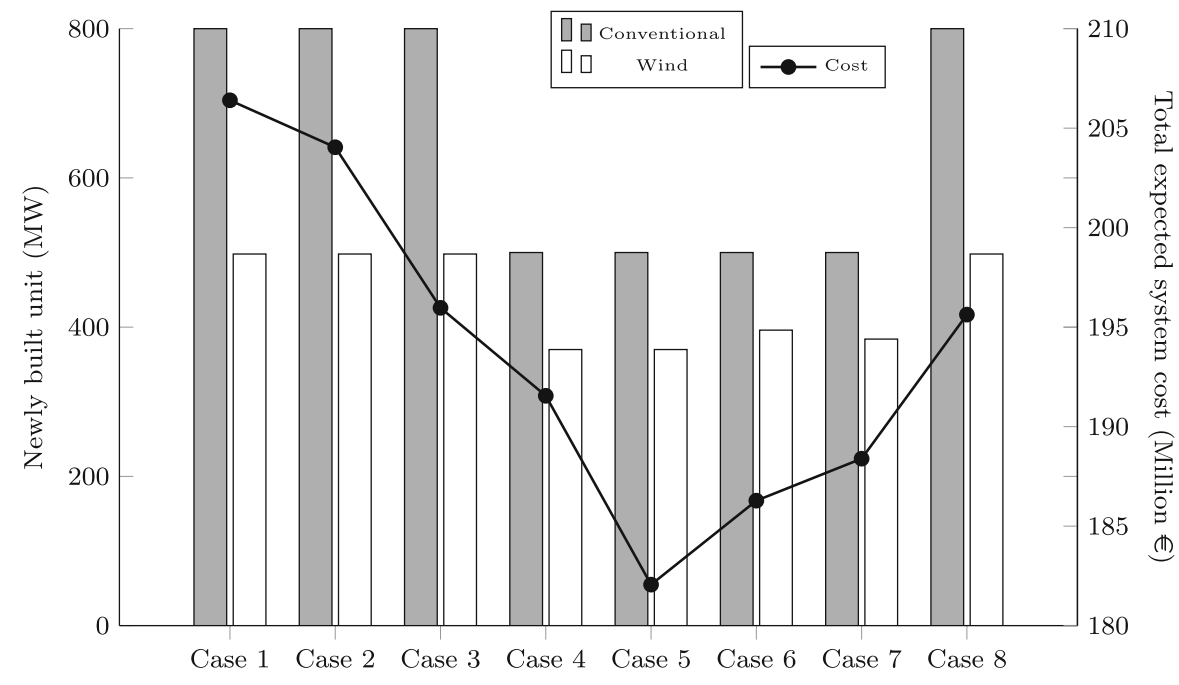

Fig. 7 Six-node test system: GEP decisions (newly built units and system cost) obtained for different cases. Case 1: full UC (benchmark), Case 2: relaxed start-up and shut-down costs, Case 3: relaxed minimum level of production, Case 4: relaxed ramping limits, Case 5: no UC constraints, Case 6: enforced start-up and shut-down costs only, Case 7: enforced minimum level of production only, Case 8: enforced ramp rates only

estimation. The next four subsections provide more details on the impact of each UC constraint on expansion decisions.

\subsubsection{Generation expansion planning model without unit commitment constraints}

Neglecting all UC constraints in the GEP decision-making in Case 5 leads to the underestimation of expected total system cost as well as a lack of investment in renewable and conventional capacity. A simple GEP model which ignores operational details underestimates the system need for flexibility and hence leads to a suboptimal capacity investment decision and generation mix. The flexibility provided through load shedding and wind curtailment is assumed to not be necessary and the need for reserve deployment in real-time operation is underrated.

The costs of neglecting the UC constraints could be quantified by enforcing the investment decisions resulted from the model with relaxed UC constraints (Case 5) within the original GEP model (Case 1) considering all UC constraints. However, in this instance, the investment decision of Case 5 leads to infeasibility of the original model. The infeasibility derives from the requirement that demand and production need to be balanced properly in the day-ahead stage of the model, and load shedding is not allowed before the real-time operation planning stage. This suggests that in the short-run system security is endangered and in the long-term system adequacy issues will arise by omitting UC constraints in GEP decision-making problem. 


\subsubsection{Impact of start-up and shut-down costs}

The start-up cost $c_{g, d, h}^{\mathrm{SU}}$ and shut-down $\operatorname{cost} c_{g, d, h}^{\mathrm{SD}}$ are included in the master problem's objective function (8a) and also in the upper and lower bounds (7) and (8a). In Case 2 omitting $c_{g, d, h}^{\mathrm{SU}}$ and $c_{g, d, h}^{\mathrm{SD}}$ causes no error of the investment decision and only a small error of underestimating the expected total system cost, partly caused by neglecting the total start-up cost of $€ 317,232$ from the original model in Case 1 . Moreover, more shut downs and start ups occur for generation units $g 2, g 6, g 7$, and $g 10$ when neglecting start-up and shut-down costs.

On the other hand, in Case 6 enforcing start-up and shut-down costs as the only UC constraint, while omitting minimum production and ramp rate limits, leads to a significant underestimation of the total expected cost as well as of the necessary investment in new renewable and conventional capacity. Furthermore, wind spillage does not occur and also the flexibility provided through load shedding is only sparsely used. The flexibility of generation units is overestimated when neglecting the hourto-hour ramp rate limitations and minimum output requirements. The modelling of start-up and shut-down costs alone cannot provide enough operational detail to reach the optimal generation mix providing sufficient flexibility.

\subsubsection{Impact of minimum production level of conventional generation units}

The minimum output level constraints are relaxed in Case 3 by setting the lower bounds on production in (3b) and (3c) for the day-ahead scheduling and in (4e) and (4f) for the real-time operation to zero. This relaxation leads to the correct investment decisions, but underestimates the total expected cost of the system. Furthermore, this relaxation leads to a reduced power adjustment by conventional generation units, wind spillage and load shedding due to the increased flexibility assumed to be provided by generation units producing below the minimum output level.

On the other hand, enforcing minimum production limits while neglecting start-up and shut-down costs and ramp rates (Case 7) leads to an underestimation of capacity investments, total cost, and the necessity for wind curtailment under some scenarios.

\subsubsection{Impact of ramp rate limits}

In Case 4 omitting equations (3f)-(3i) and (4g)-(4j) for the ramp rate limitation of existing and candidate generation units in the first and second stages leads to a large error in GEP decisions of conventional and renewable candidates. Furthermore, this relaxation overestimates the flexibility of conventional generation units in the realtime stage. Therefore, load shedding in Case 4 is reduced compared to Case 1, and wind spillage is zero.

On the contrary, Case 8 considering ramp limits while omitting start-up and shutdown costs and production limits leads to the correct investment decision and a slight underestimation of the expected system cost.

The results of Cases 4 and 8 imply that the ramp rate limits are the most important UC constraints in this specific case study to be included in the GEP problem. 


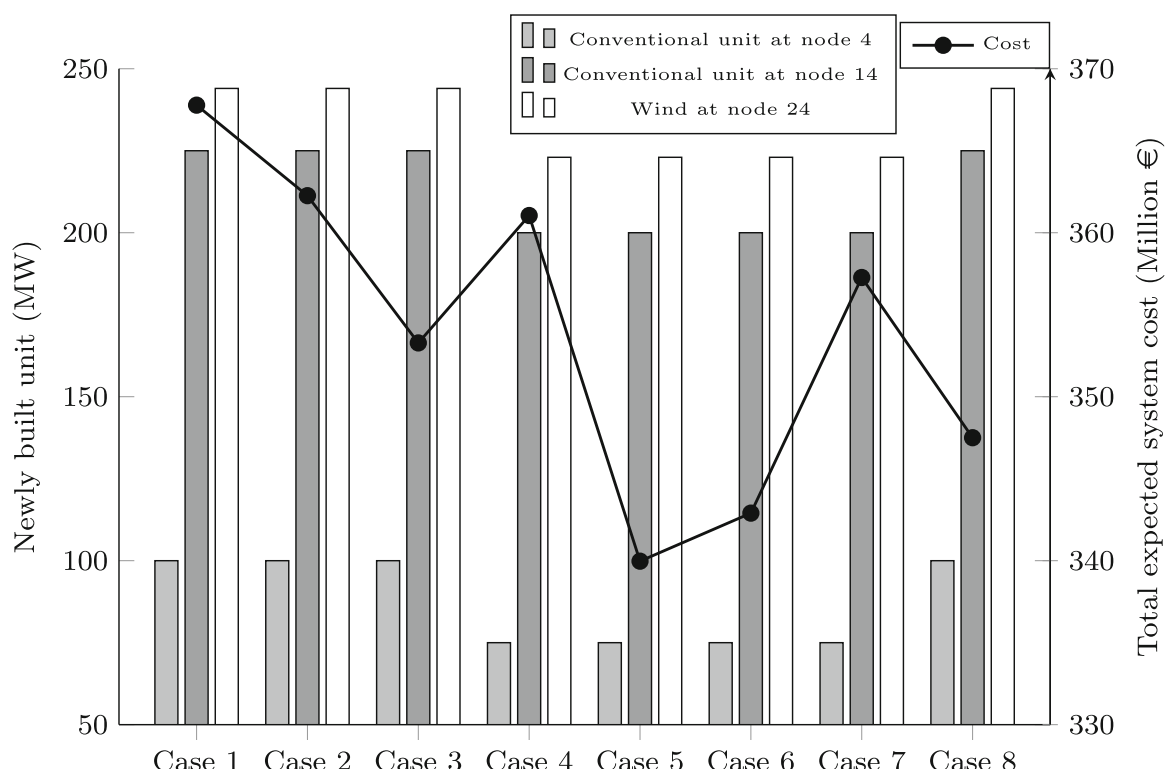

Fig. 8 IEEE 24-node RTS: GEP decisions (newly built units and system cost) obtained for different cases. Case 1: full UC (benchmark), Case 2: relaxed start-up and shut-down costs, Case 3: relaxed minimum level of production, Case 4: relaxed ramping limits, Case 5: no UC constraints, Case 6: enforced start-up and shut-down costs only, Case 7: enforced minimum level of production only, Case 8: enforced ramp rates only

\subsection{IEEE 24-node reliability test system: numerical results}

Next, we present numerical results for a larger case study based on an updated version of the IEEE 24-node reliability test system [37]. We consider five candidate conventional generation units to be built at nodes $4,8,10,14$, and 20 , each with five expansion options. In addition, we consider two candidate wind farms at nodes 19 and 24. According to the optimal GEP results obtained for a case with 100 scenarios and 10 representative days, the total new wind capacity of 1114 MW (including 870 MW at node 19 and 244 MW at node 24) as well as the total conventional capacity of $925 \mathrm{MW}$ are installed. These new conventional units have capacities of 100, 150, 200, 225, and $250 \mathrm{MW}$, with the minimum production levels of 50, 50, 50, 20, and 10 $\mathrm{MW}$, and ramp rates of 75, 100, 150, 150 and $150 \mathrm{MW} / \mathrm{h}$, respectively.

Similar to Sect. 5.2, the same eight cases in Table 3 are used to analyze the GEP decisions in the 24-node system, whose results are summarized in Fig. 8. Across all cases, the investment decisions at nodes 8, 10, 19, and 20 are unchanged. However, the investment decisions in newly built conventional and wind units at nodes 4, 14, and 20 depend on the relaxation or enforcement of UC constraints, leading to varying total expected system cost. Case 1 (as the benchmark) enforces all UC constraints, which obtains the most accurate investment decisions and the total expected system cost. The relaxation of start-up and shut-down costs (Case 2) or minimum production levels (Case 3) results in identical generation expansion planning decisions to 
those in Case 1, while underestimating the expected system cost. Relaxing the hourly ramping constraints (Case 4) causes the underestimation of both system cost and new conventional and renewable capacity required. Incorporating only ramping constraints (Case 8) obtains accurate investment decisions, while underestimating the system cost. Neglecting all UC constraints (Case 5) or only considering the start-up and shut-down costs of units (Case 6) or minimum production levels (Case 7) leads to inaccurate investment decisions and system cost underestimation.

The results from both case studies imply that UC constraints may impact the optimal generation portfolio mix. Moreover, neglecting these constraints may lead to erroneous investment decisions. Within these case studies, ramp rate limitations are shown to have the most impact, while start-up and shut-down costs and minimum production requirements seem to be less influential. However, one needs to note that these results are system specific. Nonetheless, the proposed model serves as a tool for evaluating the impact of UC constraints on capacity expansion decisions in various systems.

\subsection{Performance validation of Benders' decomposition}

We verify the well-functioning of Benders' algorithm through a numerical analysis. To be able to compare the results of original and decomposed models, we first reduce the number of scenarios to make the original model computationally tractable. We consider a case with ten representative days and ten scenarios. Our results given in Table 4 show that both direct non-decomposed and decomposed solution techniques (either using uni- or multi-cut algorithms) end up to the same investment decisions for the six-node test system. In addition, the multi-cut Benders' decomposition algorithm converges faster (10 iterations) with respect to the uni-cut one (29 iterations). This numerical analysis confirms the well-functioning of the proposed decomposition algorithm. Table 5 verifies the solution performance of the multi-cut Benders' decomposition for the IEEE 24-node reliability test system.

\subsection{Computational performance}

We solve the proposed model on an Intel CoreTM i5-5200U with two processors clocking at 2.70 GHz and $4 \mathrm{~GB}$ of RAM using CPLEX 12.6.3.0 under GAMS 24.7.4 solved with an optimality gap tolerance of $1 \%$. The predefined tolerance for Benders' convergence is $\varepsilon=0.5 \%$ of master problem's objective function obtained in each iteration. Table 6 gives the CPU time required to solve the original non-decomposed problem for both six- and 24-node case studies under different number of representative days and scenarios. As expected, it quickly becomes computationally intractable by adding more days and scenarios.

The proposed Benders' algorithm allows decomposing the original model by representative day and scenario. Therefore, it decomposes this case study to 1000 subproblems (100 scenarios and 10 representative days). The scale of each single subproblem is considerably smaller than the original non-decomposed GEP problem. However, the size and complexity of the master problem including Benders' cuts increase significantly over iterations. Table 7 illustrates the computational per- 
Table 4 Six-node test system: comparison of the results obtained from original and decomposed models for a case with ten representative days and ten scenarios

\begin{tabular}{|c|c|c|c|}
\hline Solution method & $\begin{array}{l}\text { Non-decomposed } \\
\text { model }\end{array}$ & $\begin{array}{l}\text { Decomposed model } \\
\text { with uni-cut algorithm }\end{array}$ & $\begin{array}{l}\text { Decomposed model } \\
\text { with multi-cut algorithm }\end{array}$ \\
\hline Optimality gap tolerance & $1 \%$ & $1 \%$ & $1 \%$ \\
\hline Convergence tolerance $\varepsilon$ & - & $0.5 \%$ & $0.5 \%$ \\
\hline Number of iterations & - & 29 & 10 \\
\hline $\begin{array}{l}\text { Total expected system cost } \\
{[\text { Million } €]}\end{array}$ & 205.7 & $\begin{array}{l}204.9 \\
(-0.3 \%)\end{array}$ & $\begin{array}{l}207.9 \\
(+1 \%)\end{array}$ \\
\hline $\begin{array}{l}\text { Additional wind capacity } \\
\text { (MW) }\end{array}$ & 497.7 & 497.7 & 497.7 \\
\hline $\begin{array}{l}\text { Additional conventional } \\
\text { capacity (MW) }\end{array}$ & 800 & 800 & 800 \\
\hline $\begin{array}{l}\text { Minimum production of } \\
\text { additional generator } \\
\text { (MW) }\end{array}$ & 200 & 200 & 200 \\
\hline
\end{tabular}

Table 5 IEEE 24-node RTS: comparison of the results obtained from original and decomposed models for a case with ten representative days and ten scenarios

\begin{tabular}{llll}
\hline Solution method & $\begin{array}{l}\text { Non-decomposed } \\
\text { model }\end{array}$ & $\begin{array}{l}\text { Decomposed model } \\
\text { with uni-cut algorithm }\end{array}$ & $\begin{array}{l}\text { Decomposed model with } \\
\text { multi-cut algorithm }\end{array}$ \\
\hline $\begin{array}{l}\text { Optimality gap } \\
\text { tolerance }\end{array}$ & $1 \%$ & $1 \%$ & $1 \%$ \\
$\begin{array}{c}\text { Convergence } \\
\text { tolerance } \varepsilon\end{array}$ & - & $0.5 \%$ & $0.5 \%$ \\
$\begin{array}{l}\text { Number of iterations } \\
\begin{array}{c}\text { Total expected system } \\
\text { cost }\end{array}\end{array}$ & - & 23 & 5 \\
$\begin{array}{l}\text { [Million } € \text { ] } \\
\begin{array}{l}\text { Additional wind } \\
\text { capacity (MW) }\end{array}\end{array}$ & 237 & $365.2(+0.5 \%)$ & $370.2(-0.7 \%)$ \\
$\begin{array}{c}\text { Additional } \\
\text { conventional }\end{array}$ & 325 & 237 & $244(+3 \%)$ \\
capacity (MW) & & 325 & 325 \\
\hline
\end{tabular}

formance of Benders' algorithm. The subproblems are of small size, while the master problem becomes more complex with increasing number of iterations due to multiple cuts added in each iteration. Note that a large number of representative days might still be computationally difficult even for the decomposed model, since the size and complexity of the master problem increase significantly with the number of days.

\section{Conclusions}

Many of the most commonly used GEP models have been formulated in a way that neglects the UC constraints of conventional units. The integration of variable renew- 
Table 6 Computational considerations corresponding to the direct non-decomposed solution method for both six- and 24-node case studies

\begin{tabular}{llcccccr}
\hline System & $\begin{array}{l}\text { \# Representative } \\
\text { days }\end{array}$ & \# Scenarios & $\begin{array}{l}\text { \# All } \\
\text { variables }\end{array}$ & $\begin{array}{l}\text { \# Binary } \\
\text { variables }\end{array}$ & \# Equations & Gap (\%) & $\begin{array}{r}\text { CPU } \\
\text { time (s) }\end{array}$ \\
\hline Six-node & 10 & 10 & 105,136 & 2,410 & 214,808 & 0.01 & 269.2 \\
& 10 & 20 & 196,336 & 2,410 & 404,408 & 0.01 & 6027.9 \\
& 15 & 10 & 157,696 & 3,610 & 322,208 & 0.01 & 6267.9 \\
& 10 & 50 & 469,936 & 2,410 & 973,208 & Intractable \\
24-node & 10 & 100 & 925,936 & 2,410 & $1,921,208$ & Intractable \\
\hline
\end{tabular}

Table 7 Computational considerations of the proposed multi-cut Benders' decomposition

\begin{tabular}{lllllll}
\hline System & $\begin{array}{l}\text { \# Repr. } \\
\text { days }\end{array}$ & \# Scen. & CPU time (s) & & \\
\cline { 3 - 7 } & & & $\begin{array}{l}\text { Subproblem } \\
\text { average }\end{array}$ & $\begin{array}{l}\text { Initial } \\
\text { master } \\
\text { problem }\end{array}$ & $\begin{array}{l}\text { Master problem in } \\
\text { last iteration }\end{array}$ & \# Iterations \\
\hline Six-node & 10 & 100 & 0.1 & 3.3 & 103 & 7 \\
24-node & 10 & 100 & 0.1 & 6 & 86 & 5 \\
\hline
\end{tabular}

able power has consequences for the effectiveness of existing GEP models. Where UC characteristics are not accounted for in these expansion models, changes in how units are committed and dispatched cannot be anticipated, leading to portfolios unsuited for variability.

This paper introduces an efficient method to include UC constraints into a GEP model, exploring the impacts of these operational details within a two-stage planning model under wind uncertainty. This paper also seeks to determine which UC constraint is more effective in the generation planning problems for systems with high wind power penetration, and provides critical implications to decision-makers relating to the capacity expansion planning. The illustrated case studies demonstrates that the incorporation of UC constraints affects the expansion decisions for an optimal future capacity mix. Within these specific case studies, the ramp rate limit of units has the largest impact on the GEP decisions, whereas the minimum output level and start-up and shut-down constraints are less influential. Therefore, the elimination of ramping limits in our case studies is the most distorting simplification.

Other UC constraints which can be modeled for future analysis are minimum upand down-times, or the start up of fast-start units in real-time. Moreover, the impact of active power losses, maintenance and fixed costs may be analyzed further on. In addition, the joint generation and transmission expansion planning problem may be the topic of future research. A further extension of the proposed method could be an implementation of multi-stage investment using relevant decomposition techniques, e.g., stochastic dual dynamic programming (SDDP). 
Acknowledgements We thank Amin Nasri for fruitful discussion about the initial model. We also thank Peter Meibom and David Wozabal for constructive feedback.

\section{References}

1. Belgian Transmission System Operator, Elia, Wind-power generation data. http://www.elia.be/en/griddata/power-generation/wind-power. Accessed 1 Apr 2017

2. Energy, transport and environment indicators, Annual report by Statistical Office of the European Union (Eurostat), 2016. http://ec.europa.eu/eurostat/documents/3217494/7731525/KS-DK-16-001EN-N.pdf/cc2b4de7-146c-4254-9521-dcbd6e6fafa6. Accessed 20 Nov 2018

3. PJM Market, Markets and Operations. Energy Market. Day Ahead Demand Bid. http://www.pjm.com/ markets-and-operations/energy.aspx. Accessed 1 Apr 2017

4. Batlle, C., Rodilla, P.: An enhanced screening curves method for considering thermal cycling operation costs in generation expansion planning. IEEE Trans. Power Syst. 28(4), 3683-3691 (2013)

5. Benders, J.F.: Partitioning procedures for solving mixed-variables programming problems. Numer. Math. 4(1), 238-252 (1962)

6. Birge, J.R., Louveaux, F.V.: A multicut algorithm for two-stage stochastic linear programs. Eur. J. Oper. Res. 34(3), 384-392 (1988)

7. Bruno, S., Ahmed, S., Shapiro, A., Street, A.: Risk neutral and risk averse approaches to multistage renewable investment planning under uncertainty. Eur. J. Oper. Res. 250(3), 979-989 (2016)

8. Conejo, A.J., Baringo, L., Kazempour, S.J., Siddiqui, A.S.: Investment in Electricity Generation and Transmission: Decision Making Under Uncertainty. Springer, Berlin (2016)

9. Conejo, A.J., Carrión, M., Morales, J.M.: Decision Making Under Uncertainty in Electricity Markets. International Series in Operations Research and Management Science. Springer, New York (2010)

10. Conejo, A.J., Castillo, E., Minguez, R., García-Bertrand, R.: Decomposition Techniques in Mathematical Programming: Engineering and Science Applications. Springer, Berlin (2006)

11. Connolly, D., Lund, H., Mathiesen, B.: Smart energy Europe: the technical and economic impact of one potential $100 \%$ renewable energy scenario for the European Union. Renew. Sustain. Energy Rev. 60, 1634-1653 (2016)

12. Dehghan, S., Amjady, N., Kazemi, A.: Two-stage robust generation expansion planning: a mixed integer linear programming model. IEEE Trans. Power Syst. 29(2), 584-597 (2014)

13. Flores, J.R., Montagna, J.M., Vecchietti, A.: An optimization approach for long term investments planning in energy. Appl. Energy 122, 162-178 (2014)

14. Flores-Quiroz, A., Palma-Behnke, R., Zakeri, G., Moreno, R.: A column generation approach for solving generation expansion planning problems with high renewable energy penetration. Electr. Power Syst. Res. 136, 232-241 (2016)

15. Gil, J., Caballero, A., Conejo, A.J.: Power cycling: CCGTs: the critical link between the electricity and natural gas markets. IEEE Power Energy Magz. 12(6), 40-48 (2014)

16. Hua, B., Baldick, R., Wang, J.: Representing operational flexibility in generation expansion planning through convex relaxation of unit commitment. IEEE Trans. Power Syst. 33(2), 2272-2281 (2018)

17. Jin, S., Botterud, A., Ryan, S.M.: Temporal versus stochastic granularity in thermal generation capacity planning with wind power. IEEE Trans. Power Syst. 29(5), 2033-2041 (2014)

18. Jin, S., Ryan, S.M., Watson, J.P., Woodruff, D.L.: Modeling and solving a large-scale generation expansion planning problem under uncertainty. Energy Syst. 2(3-4), 209-242 (2011)

19. Johnson, E.P.: The cost of carbon dioxide abatement from state renewable portfolio standards. Resour. Energy Econ. 36(2), 332-350 (2014)

20. de Jonghe, C., Delarue, E., Belmans, R., d'Haeseleer, W.: Determining optimal electricity technology mix with high level of wind power penetration. Appl. Energy 88(6), 2231-2238 (2011)

21. de Jonghe, C., Hobbs, B.F., Belmans, R.: Optimal generation mix with short-term demand response and wind penetration. IEEE Trans. Power Syst. 27(2), 830-839 (2012)

22. Kamalinia, S., Shahidehpour, M.: Generation expansion planning in wind-thermal power systems. IET Gen. Transmission Distrib. 4(8), 940 (2010)

23. Kazempour, J., Hobbs, B.F.: Value of flexible resources, virtual bidding, and self-scheduling in twosettlement electricity markets with wind generation - part II: ISO models and application. IEEE Trans. Power Syst. 33(1), 760-770 (2018) 
24. Kazempour, S.J., Conejo, A.J., Ruiz, C.: Strategic generation investment using a complementarity approach. IEEE Trans. Power Syst. 26(2), 940-948 (2011)

25. Koltsaklis, N.E., Georgiadis, M.C.: A multi-period, multi-regional generation expansion planning model incorporating unit commitment constraints. Appl. Energy 158, 310-331 (2015)

26. Koltsaklis, N.E., Liu, P., Georgiadis, M.C.: An integrated stochastic multi-regional long-term energy planning model incorporating autonomous power systems and demand response. Energy 82, 865-888 (2015)

27. Liu, Y., Sioshansi, R., Conejo, A.J.: Hierarchical clustering to find representative operating periods for capacity-expansion modeling. IEEE Trans. Power Syst. 33(3), 3029-3039 (2018)

28. Merrick, J.H.: On representation of temporal variability in electricity capacity planning models. Energy Econ. 59, 261-274 (2016)

29. Morales, J.M., Conejo, A.J., Pérez-Ruiz, J.: Economic valuation of reserves in power systems with high penetration of wind power. IEEE Trans. Power Syst. 24(2), 900-910 (2009)

30. Munoz, F.D., Hobbs, B.F., Watson, J.P.: New bounding and decomposition approaches for MILP investment problems: multi-area transmission and generation planning under policy constraints. Eur. J. Oper. Res. 248(3), 888-898 (2016)

31. Munoz, F.D., van der Weijde, A.H., Hobbs, B.F., Watson, J.P.: Does risk aversion affect transmission and generation planning? A Western North America case study. Energy Econ. 64, 213-225 (2017)

32. Murphy, F.H., Smeers, Y.: Generation capacity expansion in imperfectly competitive restructured electricity markets. Oper. Res. 53(4), 646-661 (2005)

33. Murphy, J.: Benders, Nested Benders and Stochastic Programming: An Intuitive Introduction. Cambridge University Engineering Department Technical Report (2013). arxiv:1312.3158

34. Nasri, A., Kazempour, S.J., Conejo, A.J., Ghandhari, M.: Network-constrained AC unit commitment under uncertainty: a Benders decomposition approach. IEEE Trans. Power Syst. 31(1), 412-422 (2016)

35. Nogales, A., Wogrin, S., Centeno, E.: Impact of technical operational details on generation expansion in oligopolistic power markets. IET Gen. Transmission Distrib. 10(9), 2118-2126 (2016)

36. Nweke, C.I., Leanez, F., Drayton, G.R., Kolhe, M.: Benefits of chronological optimization in capacity planning for electricity markets. In: IEEE power system technology (POWERCON), pp. 1-6 (2012)

37. Ordoudis, C., Pinson, P., Morales, J.M., Zugno, M.: An updated version of the IEEE RTS 24-bus system for electricity market and power system operation studies. Technical University of Denmark (2016)

38. Ostrowski, J., Anjos, M.F., Vannelli, A.: Tight mixed integer linear programming formulations for the unit commitment problem. IEEE Trans. Power Syst. 27(1), 39-46 (2012)

39. Palmer, K., Burtraw, D.: Cost-effectiveness of renewable electricity policies. Energy Econ. 27(6), 873-894 (2005)

40. Palmintier, B.: Flexibility in generation planning: Identifying key operating constraints. In: Power systems computation conference (PSCC), 2014, pp. 1-7. IEEE (2014)

41. Palmintier, B., Webster, M.: Impact of unit commitment constraints on generation expansion planning with renewables. In: IEEE power and energy society general meeting, pp. 1-7 (2011)

42. Palmintier, B.S., Webster, M.D.: Impact of operational flexibility on electricity generation planning with renewable and carbon targets. IEEE Trans. Sustain. Energy 7(2), 672-684 (2016)

43. Papavasiliou, A., Oren, S.S., O’Neill, R.P.: Reserve requirements for wind power integration: a scenario-based stochastic programming framework. IEEE Trans. Power Syst. 26(4), 2197-2206 (2011)

44. Pina, A., Silva, C.A., Ferrão, P.: High-resolution modeling framework for planning electricity systems with high penetration of renewables. Appl. Energy 112, 215-223 (2013)

45. Pineda, S., Morales, J.M., Boomsma, T.K.: Impact of forecast errors on expansion planning of power systems with a renewables target. Eur. J. Oper. Res. 248(3), 1113-1122 (2016)

46. Pinson, P., Mitridati, L., Ordoudis, C., Ostergaard, J.: Towards fully renewable energy systems: experience and trends in Denmark. CSEE J. Power Energy Syst. 3(1), 26-35 (2017)

47. Poncelet, K., Delarue, E., Six, D., Duerinck, J., d'Haeseleer, W.: Impact of the level of temporal and operational detail in energy-system planning models. Appl. Energy 162, 631-643 (2016)

48. Poncelet, K., Höschle, H., Delarue, E., Virag, A., d'Haeseleer, W.: Selecting representative days for capturing the implications of integrating intermittent renewables in generation expansion planning problems. IEEE Trans. Power Syst. 32(3), 1936-1948 (2017)

49. Puga, J.N.: The importance of combined cycle generating plants in integrating large levels of wind power generation. Electr. J. 23(7), 33-44 (2010) 
50. Schwele, A., Kazempour, J., Pinson, P.: Electronic companion for paper: do unit commitment constraints affect generation expansion planning? A scalable model (2017). https://doi.org/10.5281/ zenodo. 1116956

51. Shortt, A., Kiviluoma, J., O’Malley, M.: Accommodating variability in generation planning. IEEE Trans. Power Syst. 28(1), 158-169 (2013)

52. Shortt, A., O’Malley, M.: Impact of variable generation in generation resource planning models. In: IEEE power and energy society general meeting, pp. 1-6 (2010)

53. Sifuentes, W.S., Vargas, A.: Hydrothermal scheduling using Benders decomposition: accelerating techniques. IEEE Trans. Power Syst. 22(3), 1351-1359 (2007)

54. Singh, K.J., Philpott, A.B., Wood, R.K.: Dantzig-Wolfe decomposition for solving multistage stochastic capacity-planning problems. Oper. Res. 57(5), 1271-1286 (2009)

55. Skar, C., Doorman, G., Tomasgard, A.: Large-scale power system planning using enhanced Benders decomposition. In: Power systems computation conference (PSCC), pp. 1-7 (2014)

56. Staffell, I., Green, R.: Is there still merit in the merit order stack? The impact of dynamic constraints on optimal plant mix. IEEE Trans. Power Syst. 31(1), 43-53 (2016)

57. Trukhanov, S., Ntaimo, L., Schaefer, A.: Adaptive multicut aggregation for two-stage stochastic linear programs with recourse. Eur. J. Oper. Res. 206(2), 395-406 (2010)

58. Upton Jr., G.B., Snyder, B.F.: Funding renewable energy: an analysis of renewable portfolio standards. Energy Econ. 66, 205-216 (2017)

59. van Stiphout, A., de Vos, K., Deconinck, G.: The impact of operating reserves on investment planning of renewable power systems. IEEE Trans. Power Syst. 32(1), 378-388 (2017)

60. Villumsen, J.C., Bronmo, G., Philpott, A.B.: Line capacity expansion and transmission switching in power systems with large-scale wind power. IEEE Trans. Power Syst. 28(2), 731-739 (2013)

61. Westner, G., Madlener, R.: Investment in new power generation under uncertainty: benefits of CHP vs. condensing plants in a copula-based analysis. Energy Econ. 34(1), 31-44 (2012)

62. Wolf, C., Koberstein, A.: Dynamic sequencing and cut consolidation for the parallel hybrid-cut nested L-shaped method. Eur. J. Oper. Res. 230(1), 143-156 (2013)

63. You, F., Grossmann, I.E.: Multicut Benders decomposition algorithm for process supply chain planning under uncertainty. Ann. Oper. Res. 210(1), 191-211 (2013)

64. Zhang, Q., Cremer, J.L., Grossmann, I.E., Sundaramoorthy, A., Pinto, J.M.: Risk-based integrated production scheduling and electricity procurement for continuous power-intensive processes. Comput. Chem. Eng. 86, 90-105 (2016)

65. Zverovich, V., Fábián, C.I., Ellison, E.F., Mitra, G.: A computational study of a solver system for processing two-stage stochastic LPs with enhanced Benders decomposition. Math. Program. Comput. 4(3), 211-238 (2012)

Publisher's Note Springer Nature remains neutral with regard to jurisdictional claims in published maps and institutional affiliations.

\section{Affiliations}

\section{Anna Schwele ${ }^{1}$ (D) Jalal Kazempour ${ }^{1}$ (D) $\cdot$ Pierre Pinson $^{1}$ (D}

Jalal Kazempour

seykaz@elektro.dtu.dk

Pierre Pinson

ppin@elektro.dtu.dk

1 Department of Electrical Engineering, Technical University of Denmark, 2800 Kongens Lyngby, Denmark 